ESAIM: PROCEEDINGS AND SURVEYS, 2020, Vol. 68, p. 123-152

Hervé Cardot \& Pierre Calka

\title{
INDIVIDUAL-BASED MODELS UNDER VARIOUS TIME-SCALES
}

\author{
AurÉLIEN VELLERET ${ }^{1}$
}

\begin{abstract}
This article is a presentation of specific recent results describing scaling limits of individualbased models. Thanks to them, we wish to relate the time-scales typical of demographic dynamics and natural selection to the parameters of the individual-based models. Although these results are by no means exhaustive, both on the mathematical and the biological level, they complement each other. Indeed, they provide a viewpoint for many classical time-scales. Namely, they encompass the timescale typical of the life-expectancy of a single individual, the longer one wherein a population can be characterized through its demographic dynamics, and at least four interconnected ones wherein selection occurs. The limiting behavior is generally deterministic. Yet, since there are selective effects on randomness in the history of lineages, probability theory is shown to be a key factor in understanding the results. Besides, randomness can be maintained in the limiting dynamics, for instance to model rare mutations fixing in the population.

Résumé. Les résultats récents présentés dans cet article décrivent des limites d'échelles de modèles individus-centrés. Grâce à eux, nous allons mettre en lumière les différentes échelles de temps caractéristiques des variations démographiques et de la sélection naturelle. L'objectif n'est pas d'être exhaustif, tant au niveau mathématique que biologique. Néanmoins, ces résultats sont très complémentaires les uns des autres. Ils fournissent un aperçu des principales échelles de temps d'intérêt. Ils englobent notamment l'échelle de temps de la vie d'un individu, celle plus longue où la population peut être décrite comme une entité avec ses caractéristiques propres qui dirigent les dynamiques démographiques, et au moins quatre autres échelles imbriquées sur lesquelles la sélection joue un rôle. La dynamique limite est généralement déterministe. Pour autant, puisque l'action de la sélection se base sur l'aléa présent dans l'histoire des individus, les probabilités apparaissent comme un élément clé pour comprendre ces résultats. Par ailleurs, la stochasticité peut aussi être conservée à la limite, par exemple pour modéliser de rares événements de fixation de mutations.
\end{abstract}

\section{INTRODUCTION}

The origin of this paper is the session "Stochastic Processes and Biology" during the conference Journées MAS in Dijon at the end of August 2018. Four talks were presented, with a variety of methods and probabilistic approaches. Their common feature, that motivates this presentation, is the fact that they start from similar Individual-Based Models (IBM) and justify an asymptotic behavior as both the population size and the observation time are large. The time-scales wherein these observations can be obtained are nonetheless different, yet interconnected. The structure of the paper aims both at presenting the principal objects of the four talks of the session and at reflecting this increasing sequence of time-scales involved in adaptation of populations.

1 Aix-Marseille Universite, CNRS, Centrale Marseille, I2M, UMR 737313453 Marseille, France, email : aurelien.velleret@univamu.fr

(C) EDP Sciences, SMAI 2020

This is an Open Access article distributed under the terms of the Creative Commons Attribution License (http://creativecommons.org/licenses/by/4.0), which permits unrestricted use, distribution, and reproduction in any medium, provided the original work is properly cited.

Article published online by EDP Sciences and available at ittps://www.esaim-proc.org or ittps://doi.org/10.1051/proc/202068007 
For more than fifteen years, there has been a significant activity around a new probabilistic framework for mathematical modeling of ecology, population genetics and trait evolution. Traits are any features of the individuals whose transmission the modeler is interested in, and we refer to the beginning of Chapter 1 for some classical examples. They may vary during the life-time of the individuals, at births or only at rare mutation events. The population is described by the distribution of its traits, from which one may for instance deduce its growth rate. Any effect of natural selection should depend on these traits and affect their distribution.

The IBM are a priori the best formulation for validation of a macroscopic model of population dynamics. These processes detail at individual level births, deaths and interactions in the population. The description may also specify migration patterns, aging or competition for resources between individuals. This setting is clearly the closest to actual simulations designed by computational biologists to validate theoretical models, or simply some predictions. In this view, such setting is also the one with the least number of simplifying assumptions. It is also certainly the one where calculations of probabilities of events are the most complicated. One of the first aims of such probabilistic modeling has actually been to connect these individual-based models and simulations to much simpler systems of Ordinary Differential Equations (ODE) or Partial Differential Equation (PDE) models.

To describe natural selection on heritable traits, systems of ODE usually provide the most classical models to simply express their effects. They have been refined by introducing PDE to deal with a continuum of traits in populations. Thanks to these models, one can cover most of actual mathematical models of ecology, population genetics and character evolution. Yet, this deterministic approach does not take into account the variability observed when reproducing similar experiments. A part of this variability is due to external perturbations. Stochastic Differential Equations (SDE) have been introduced to model these effects. They usually assume that the parameters appearing in the systems of ODE / PDE are themselves subject to stochasticity. The other main component of this noise is internal and inherent to the discreteness of the system : selection between traits emerges through competition between a finite number of individuals. Taking this component into account is the purpose of these IBM.

To justify simpler models from the IBM, the usual assumption is that populations are large enough so that the law of large numbers makes random fluctuations negligible. Given the finite population sizes ecologists are interested in, an elementary description may well be accurately designed while a finer structuring of the population is much more demanding.

It is rather natural for mathematicians to try to describe simplified behavior through an asymptotic on large time-scales (i.e. for large number of generations). It is at the core of Darwin's theory of natural selection to assume a separation of time-scale between apparently incidental randomness among contemporary individuals and the evolution of traits, and even of species. The goal is surely to obtain exact results of convergence to a non-trivial process for the new dynamics in a new time-scale. It is however very challenging, and perhaps an approximation cannot be justified in this way, while still being helpful. There is also no reason to restrict ourselves purely to deterministic limiting processes : a first order of fluctuations can be inferred from central limit theorems. For instance, it may take the form of a solution to a specific SDE. Such a next order notably helps to specify the conditions of validity of the deterministic approximation.

An archetypal example is the stochastic approximation of the population size in a continuous setting (i.e. with Brownian-type fluctuations). Beyond such a result is the idea that individual events (of births or deaths) have quite a negligible impact on the population as a whole, yet randomness still occurs through varying accumulation of such effects. Thus, to obtain the convergence towards a stochastic continuous process, one needs (besides the population size going to infinity) to amplify the frequency of births and deaths also. Notably, this means that selective effects appear in a much longer time-scale than the life-expectancy of individuals (cf. in particular chapter 3 in [4]). The scale of population size provides the essential relation between the parameters at individual time-scale (on this time-scale, the birth rate is around 1) and at population level (cf. section 1). In case there is more than one parameter to adjust, analysis may be even less intuitive and may even lead to 
different behaviors associated with different scalings.

Each of the four talks given during the above-mentioned session constitutes the foundation of each of the four sections to follow. The results that we will present are taken from the referenced articles, and the reader is spared some details on the underlying assumptions. These choices and the discussions that complement these results express my personal view on the subject, and do not engage the authors of the articles. My aim while reporting these results is to highlight the variety of time-scales involved in population models. These population models are simpler to analyze and quicker to simulate than the IBM for large populations. We will thus specify how large the population sizes shall be in order to have valid estimations of individual-based models through population models.

In Section 1, we shall present the work of Hoffmann and Marguet [18]. IBM are introduced in terms of traits that structure the population and evolve in this section during the life-time of individuals. The estimation of this trait dynamics is the purpose of their paper, in which specific estimators are proposed and their accuracy evaluated. From this evaluation, we will obtain some insight into the time-scale required for different populations (with different trait dynamics) to be distinguished. The following sections are devoted to evolution and the associated models rely on traits that are much more accurately transmitted to descendants. Although this previously described variability is usually neglected in evolutionary studies (only some average effect is considered), we shall see in Section 2 that it may play a significant role when two components of selection induce competing effects. Both the strength and the time-scale of selection between two sub-populations may in fact involve the amount of a priori non-selective variability. This is one of the main conclusions that I wished to discuss based on my work in [26]. Assuming however that this variability has only a minor effect on the 'local' reproductive value of individuals and rescaling time properly, Champagnat and Henry describe in [10] the process of evolution as an almost deterministic behavior. This work and the connections to the two previous sections will be our concern in Section 3. Finally, natural selection may rely on the emergence of very rare mutations, as in Tran's talk. The associated time-scale depends on the occurrence rate and the mean effect of successful mutations (that invade the population). Among several possibilities, I have chosen to present the models from [7] and [2] in Section 4.

\section{RECOGNITION OF POPULATION TRAits FROM INDIVIDUAL DYNAMICS}

This section is devoted to the results of Hoffmann and Marguet. For simplicity, the model neglect any interaction between individuals other than transmission of specified traits from parents to offspring, what we call a model of branching population. Assuming that changing traits induce a structure on this branching population, they propose a statistical estimation of this dynamics of traits [18]. This is the right place to discuss this notion of individual-based models and how to relate individual histories to the law that governs these behaviors. It gives some insight into the number of generations involved before one can actually distinguish distinct population models. On a shorter time-scale, natural selection may favor some trajectories by mere chance. Yet those lucky behaviors should be observed with close frequencies between distinct populations. This selection shall thus hardly lead to any heritable effect. For instance, as presented in [18], any statistical estimation of traits requires a sufficiently large population history. Likewise, a long-term history is required for natural selection to favor one subpopulation over another (with different inheritance). Moreover, an initially very favorable combination of trait and environment might not be so beneficial in the long term : most descendants might be exposed later on to a much less favorable environment given their traits. This "sample size" shall thus be larger when there are strong correlations between successive generations. In cases where such correlations are sufficiently weak, Hoffmann and Marguet (cf. [18]) study how to take them into account in statistical analysis. They indicate the accuracy of some estimators in terms of the size of the genealogical tree on which observations are indexed. The focus in [18] is on the random process governing birth events, for which they propose the first statistical analysis in a structured population. 
At an individual-based level, each individual is characterized by some value $x \in \mathcal{X}$, that we call a trait. Typically $\mathcal{X} \subset \mathbb{R}^{k}$ or $\mathcal{X} \subset \mathbb{R}^{k} \times F$, where we may consider $k$ characteristics such as size, spatial position, age, the amount of certain proteins, of certain ressources, of parasites and so on, while $F$ denotes a finite (or numerable) set of classes, such as sex, eye color etc. Before the death of the individual, this trait $x$ may be constant, but it can also evolve according to some stochastic differential equation (SDE). Interaction with other individuals can then appear in parameters of this equation, possibly depending on the trait $x^{\prime}$ of the other individuals around. Death of individuals happens at a rate $d$, that may depend first on the trait $x$ of the individual, but also on the whole population and eventually its effect on the environment (as is the case in Section 3). We recall that we could include the age of the individual in the information carried by $x$, so that there is almost no restriction. Death at a fixed age is also not difficult to include in the model. Same kind of dependencies may be considered for births, and apply to the number of offspring and their states at birth. Independent exponential variables or Poisson Point Processes are thus usually a very convenient way to encode all of these events. In a nutshell, one can a priori represent with stochastic IBM models any computational model that one could design for validation of biological predictions, as soon as one can follow the individuals one by one. I refer notably to Subsection 4.2 for a more concrete description of such measure-valued process. In the following, we will focus the analysis on continuous trait-spaces, for which one can exploit the regularity of the estimated functions.

\subsection{Discussion on the assumptions in [18]}

\subsubsection{A process on an incomplete tree}

The main specificity of this statistical approach is to deal with the dependency given by the genealogy of individuals. For instance, looking at the size as a trait, the larger the mother-cell is, the larger its daughter-cells shall be. With the experiments where one follows each cell individually, one can also easily obtain the entire associated lineages. Yet, many of these cells are no longer observable because of the design of experimental processes. Although the authors do not mention it, death of some cells is likely to be included as well. The statistical analysis of the traits thus relies on data that is indexed by some tree that is "incomplete" as compared to the complete binary tree. A more detailed definition on the assumption on the tree is given below. In order to relate the accuracy of the estimators to the number of birth events, the authors consider both the cases of a bounded population size at each generation and the case of an population size expanding at a given growth rate :

Definition 1.1. Consider the complete binary tree, on which our tree will be indexed. Define the generation $n$ of this complete tree as $G_{n}$. Note that $\operatorname{Card}\left(G_{n}\right)=2^{n}$.

A regular incomplete tree is a family of subsets $U_{n}$ of the complete tree up to generation $n$ such that :

(i) the parent of any individual of $U_{n}$ is also in $U_{n}$

and (ii) for some $0 \leq \rho \leq 1: 0<\liminf _{n \rightarrow \infty} 2^{-\rho n} \operatorname{Card}\left(U_{n} \cap G_{n}\right) \leq \lim \sup _{n \rightarrow \infty} 2^{-\rho n} \operatorname{Card}\left(U_{n} \cap G_{n}\right)<\infty$.

In the case $\rho \in(0,1]$, the data characterize a growing population of cells. For instance, it corresponds to an experiment where one lets these cells duplicate freely in a rich medium. In the case $\rho=0$, one rather expects some population size at equilibrium, although it is not required here. It also corresponds to the experiments where only one cell is followed after each division (which is the case in recent experiments in a microfluidic environment : the mother-machine).

In the following results, this indexation tree is considered as given. It means that we consider the trait dynamics conditionally on the realization of the tree. Yet, the interest of the experimenter is a priori rather on intrinsic parameters without conditioning. Thus, there is an implicit assumption that the shape of the indexation tree is independent of the trait dynamics.

With a single lineage experiment, this is not really an issue. But if one has to consider natural death events while estimating variable birth rates, assuming the independence seems much more questionable. It appears a bit surprising to have a birth rate depending on the trait while the probability of giving birth in one life-span 
is independent of it. A constant death rate would be a more natural assumption than a prescribed tree. Yet, it would be much more difficult to analyze and would possibly not reflect the design of the experiment very well.

\subsubsection{Ergodicity of the trait}

The other main assumption in [18] is the very rapid convergence to the ergodic measure $\nu$ in the transmission of the trait at birth from the parent to its offspring. Assuming that $u \in U_{n} \cap G_{n}$ describes the parental index of an individual with index $v \in U_{n+1} \cap G_{n+1}$, the trait at birth $X_{v}$ of the latter is given by $Q\left(X_{u}, d x_{v}\right)$ where $X_{u}$ is the trait at birth of the parent. The convergence of the estimators is stated uniformly as long as there exist $C>0$ and a positive weight-function $V$ such that for any $m \geq 1$, and $\varphi$ satisfying $\|\varphi\|_{V}:=\sup _{x}|\varphi(x)| /[1+V(x)]<\infty$ :

$$
\left\|Q^{m}(\varphi)-\nu(\varphi)\right\|_{V} \leq C 2^{-m}\|\varphi-\nu(\varphi)\|_{V}
$$

The set of such $Q$ is denoted $\mathbb{Q}_{1 / 2}$. Note in this view that the authors choose exploit exclusively traits at birth for their statistical estimations.

\subsubsection{Definition of the stochastic process, regularity and confinement}

The model in [18] is concerned with a trait supposedly governed during the life-time of each individual by a stochastic flow of the form : $d X_{t}=b\left(X_{t}\right) d t+\sigma\left(X_{t}\right) d W_{t}$, where $W$ refers to a standard Brownian Motion, and $\left(X_{t}\right)$ evolves on $\mathcal{X} \subset \mathbb{R}^{k}$. Birth occurs at time $s$ at rate $B\left(X_{s}\right)$. At reproduction event, the authors assume that the value of the trait is distributed between two offspring with the following mechanism : given an independent r.v. $\theta$, the trait at birth of the two offspring is given respectively by $\theta y$ and $(1-\theta) y$. $\theta$ is drawn according to $\kappa(y) d y$, for some probability density function $\kappa(y)$ on $[0,1]$.

This model can for instance represent the size of the cell or the propagation of a parasite (that we take here as an archetype). The main issue is here to estimate the effect of the amount of parasites in a cell on its reproduction. Note that the individual level is the one of a given cell, and not of a given parasite, as it would be in a completely discretized model. Yet, the number of parasites is assumed to be so large that a continuous description by a random process is a well-justified simplification. The estimation is focused on the effect of these parasites on the birth rates $(B(x): x \in \mathcal{X})$ of the hosts and on the law of distribution $(\kappa(y): y \in[0,1])$ between offspring.

Assumptions are specifically designed in [18] for such a model. In a broader perspective, we rather focus on the core principles for which they are introduced and refer to [18] for the precise statements. By Assumption 2 on the drift and diffusion coefficient, the authors ensure that the trait $\mathcal{X}$ stays somewhat confined around 0, with a uniformly elliptic and non-singular diffusion. By Assumption 3 on the birth rate, they ensure some regularity in $x$, a boundedness condition on the potential explosion of births and they prevent the vanishing of these events. Finally, by Assumption 4 on the splitting of $x$ at birth between the two newborns, they ensure a lower-bounded density on the fragmentation parameter, and prevent too asymmetric partitioning. The authors mention that this assumption could probably be relaxed.

\subsection{Main results for the estimations of the Generation kernel and the birth rate}

The first step of the analysis consists in the estimation of the kernel $Q$ and its stationary distribution $\nu$. This step should be easily generalized in a broader perspective of kernels $Q$.

For any function $\psi: \mathcal{X} \times \mathcal{X} \rightarrow \mathbb{R}$, and $y \in \mathcal{X}$, consider $\psi_{\star}(y):=\sup _{x \in \mathcal{X}}|\psi(x, y)|, \psi^{\star}(x):=\sup _{y \in \mathcal{X}}|\psi(x, y)|$. Denoting by $\wedge$ the minimum, we also define for any positive measure $\rho$ on $\mathcal{X}$ :

$$
\begin{gathered}
|\psi|_{\rho}:=\int_{\mathcal{X}^{2}}|\psi(x, y)| \rho(d x) d y+\left(\int_{\mathcal{X}^{2}}|\psi(x, y)| d x d y \wedge \int_{\mathcal{X}}\left|\psi_{\star}(y)\right| d y\right) \\
\mathcal{M}_{\mathrm{U}_{n}}(\psi):=\frac{1}{\operatorname{Card}\left(\mathrm{U}_{n}^{\star}\right)} \sum_{u \in \mathrm{U}_{n}^{\star}} \psi\left(X_{u-}, X_{u}\right),
\end{gathered}
$$


where $\mathrm{U}_{n}^{\star}$ is $\mathrm{U}_{n}$ deprived from the root, and $X_{u-}, X_{u}$ denotes respectively the trait at birth of the parent of $u$ and the one of individual $u$ itself. Recall that the transition from $X_{u-}$ to $X_{u}$ is given by $Q\left(X_{u-}, d x\right)$.

Proposition 1.2. Let Assumptions 2, 3 and 4 be satisfied. Let $\mu$ be a probability measure on $\mathcal{X}$ such that $\mu\left(V^{2}\right)<\infty$. Let $\psi: \mathcal{X} \times \mathcal{X} \rightarrow \mathbb{R}$ a bounded function such that $\psi_{\star}$ is compactly supported. If $\mathrm{U}_{n}$ is a regular incomplete tree, the following estimate holds true:

$$
\mathbb{E}_{\mu}\left[\left(\mathcal{M}_{\mathrm{U}_{n}}(\psi)-\nu(Q \psi)\right)^{2}\right] \lesssim \operatorname{Card}\left(\mathrm{U}_{n}\right)^{-1}\left(\left|\psi^{2}\right|_{\mu+\nu}+\left|\psi^{\star} \psi\right|_{\mu}+\left(1+\mu\left(V^{2}\right)\right)\left|\psi_{\star}\right|_{1}|\psi|_{\nu}\right)
$$

where the symbol $\lesssim$ means up to an explicitly computable constant that depends only on supp $\left(\psi_{\star}\right)$ as long as $\mathrm{Q} \in \mathbb{Q}_{1 / 2}$. More generally, it would also depend on $Q$.

For the following theorem, we assume that the operator $Q$ has a density $(q(x, y))_{(x, y) \in \mathcal{X}^{2}}$ w.r.t. Lebesgue measure on $\mathcal{X}$, with some Hölder regularity. I refer to [18] for the exact definition of the sets $\mathbb{Q}_{1 / 2}^{\alpha, \beta}(R)$, where the value $R>0$ defines a bound on the Hölder regularity of respectively order $\alpha$ in $x$ and $\beta$ in $y$. The "1/2" refers to the property (1). Given such a regularity, the authors propose to adjust the order of the estimation kernel and explain how to choose the associated window sizes given $\left|\mathrm{U}_{n}\right|$ (for some large $n \geq 1$ ). The estimation of $q$ also depends on a threshold $\varpi_{n}$ that is to adjust. I refer to [18] for the exact definitions of the estimators $\hat{\nu}_{n}(y)$ and $\hat{q}_{n}(x, y)$ of respectively $\nu(x)$ and $q(x, y)$ (with the knowledge of $\mathrm{U}_{n}$ ).

Theorem 1.3. Let Assumptions 2, 3 and 4 be satisfied. Assume that the initial distribution $\mu$ is absolutely continuous w.r.t. the Lebesgue measure with a locally bounded density and satisfies $\mu\left(V^{2}\right)<\infty$. Let $\alpha, \beta>0$. Then, for any $\varrho$-regular incomplete tree $\mathrm{U}_{n}$ and any $R>0$,

$$
\begin{aligned}
& \sup _{Q \in \mathbb{Q}_{1 / 2}^{\alpha, \beta}(R)}\left(\mathbb{E}_{\mu}\left[\left(\hat{\nu}_{n}(y)-\nu(y)\right)^{2}\right]\right)^{1 / 2} \lesssim \operatorname{Card}\left(\mathrm{U}_{n}\right)^{-\beta /(2 \beta+1)} . \\
& \sup _{Q \in \mathbb{Q}_{1 / 2}^{\alpha, \beta}(R)}\left(\mathbb{E}_{\mu}\left[\left(\hat{q}_{n}(x, y)-q(x, y)\right)^{2}\right]\right)^{1 / 2} \lesssim \varpi_{n}^{-1} \operatorname{Card}\left(\mathrm{U}_{n}\right)^{-s(\alpha, \beta) /(2 s(\alpha, \beta)+1)}
\end{aligned}
$$

hold true, where $s(\alpha, \beta)^{-1}:=(\alpha \wedge \beta)^{-1}+\beta^{-1}$ is the effective anisotropic smoothness associated with $(\alpha, \beta)$.

Note that the rate of convergence depends on the regularity of $q$. The higher it is, the more useful information we can gather from observed transitions from vicinities of $x$ to vicinities of $y$. The accuracy of the estimators is thus better supported.

The estimation of the division rate is much more complicated. Additional assumptions are required. The study is restricted to diffusion of the trait on a compact space, so that (2) can be strengthened with a right-hand side only depending on the $L^{\infty}$-norm of $\psi$. Again, we restrict the study to $\mathrm{Q} \in \mathbb{Q}_{1 / 2}$. A parametric approach is considered, where $B$ is encoded by parameters $\vartheta \in \Theta$. The associated Fisher matrix $\Psi(\vartheta)$ is assumed to be non-singular to avoid issues of identifiabilty. They also assume some upper-bounds on the derivative of $B$ along $\vartheta$ up to the third order. Moreover, the authors prevent any degeneracy and singularities of the birth rate. Finally, this birth rate $B$ is assumed to be a globally monotone function of $\vartheta$ (uniformly in $\mathcal{X}$ ).

Theorem 1.4. Let the above-mentioned assumptions be satisfied. For every $\vartheta$ in the interior of $\Theta$, if $\mathrm{U}_{n}$ is a @-regular incomplete tree :

$$
\sqrt{\operatorname{Card}\left(\mathrm{U}_{n}\right)} \times\left(\hat{\vartheta}_{n}-\vartheta\right) \rightarrow \mathrm{N}\left(0, \Psi(\vartheta)^{-1}\right)
$$

in distribution as $n \rightarrow \infty$, where $\mathrm{N}\left(0, \Psi(\vartheta)^{-1}\right)$ denote the d-dimensional Gaussian distribution with mean 0 and covariance the inverse of $\Psi(\vartheta)$.

Note that we find in this case the classical rate of convergence with order the square root of the number of observations, as for classical Markov Chains. It hints at the fact that every observed transition from parents to offspring contributes to the estimation of $\vartheta$. 
The monotonicity condition of $B$ along $\vartheta$ seems very restrictive, especially in the case of multidimensional parameter. Relaxing this condition would be a natural direction to look at for improvement.

\section{Concluding remarks :}

As mentioned after the assumption on the genealogical tree, considering this tree as prescribed is much more convenient. The fact that the convergence results of Theorems 1.3 and 1.4 depend on $U_{n}$ only through its cardinal indicates that these results shall be quite robust concerning the specific realization of the tree.

However, it does not exclude that the estimators are slightly biased by the fact that certain transmission patterns might lead to a greater probability of survival (of the lineages). For instance, it may be beneficial to divide early, in order not to let the parasites divide for long before the next division. Traits favoring early division are thus more likely to be transmitted. This bias depends on how strongly these effects may have shaped the genealogical tree. Given that the design of the experimental process has a strong effect on the shape of the tree, including this dependency would though be very difficult and likely to introduce even more bias. Besides, the estimation of the biased birth rate may be the main interest. It only means that one infers the dynamics of a "successful" lineage, with luck playing a role in this success, as well as environmental conditions.

This issue of estimating individual parameters by looking at the population as a whole leads to similar issues of independence. Notably, the population around the focal individual can be considered as a component of its environment. One may wish that the parameters of its dynamics depend on the interaction it has with this extended environment. The models become much simpler if one can neglect the detailed interactions between the individuals, and replace them by average effects. In the limit of a large population, for a system at equilibrium, such assumption may be justified by the Law of Large Number. Indeed, the effects of the interactions are averaged, as long as they are not too local, and thus almost globally constant. In results of propagation of chaos, such property is generalized to cases where the population is not at equilibrium. I refer to Sznitman's lecture at Saint-Flour [25] for an overview on this topic. In such a limiting case, known as McKean-Vlasov equations, the law of the process itself acts on the individual dynamics, together with fluctuations specific to this individual. At equilibrium, this law is all the more stable because the population size is large. Thus, we can deal with the effects of these interactions through some hidden parameters (like an effective death or birth rate). And as for the analysis of this section, estimating them by simply looking at death and reproduction times would produce a bias if they depend on heritable factors. But this bias is presumably small if heritability is weak.

To study evolution of population traits, that is for instance $\vartheta$ if mutations could alter this value, it is very classical to assume that the population size is large. Since one usually assumes a separation of time-scales between demographic dynamics and evolutionary processes, there is clearly time for individual fluctuations to be averaged. Heritable traits can leave a sufficiently clear mark for natural selection to be effective. When the environment itself is affected by the traits of individuals, as in the next model, it might however not be so clear what an average effect would be.

\section{Selection With two LeVels}

The foundation of this section is my work on the ability of selective effects acting at a group level to compensate for those acting inside each group at an individual level [26]. For simplicity, we focus here on competition between two types in a population of fixed and large size. In real populations and IBM models, there are fluctuations in the proportion due to the inexact compensation between births and deaths events occurring with the same large rate. Biologists usually refer to it as genetic drift, and often neglect its effect in the case of large populations. One shall see however that these fluctuations may not be neutral at all in a model where two selective effects are considered : the first one favors some individuals inside their groups (individual level selection) while the other favors some groups depending on the individuals they gather (group level selection). Reducing random fluctuations inside each group strongly hinders response to selection at group level. For clarity, we shall assume that these selective effects are conflicting. For instance, one may ask if and how the 
inefficient or cheater individuals can be regulated through natural selection at this group level.

The individual-based model is taken from [20], where a formalism for group selection is introduced. All groups have the same size $n \in \mathbb{N}$. There are two types of individuals : $C$ and $D$. Type $D$ individuals have a better reproduction at the individual level ( $D$ for defectors) while type $C$ individuals are positively selected at the group level ( $C$ for cooperators). Replication and selection occur concurrently at individual and group levels according to a "nested Moran process", as introduced in [15] and recalled next. Type $C$ individuals replicate at rate $w_{I}$ and type $D$ individuals at rate $w_{I}(1+s), s \geq 0$. When an individual gives birth, another individual in the same group is selected uniformly at random to die and be replaced, so that the population size remains constant. To reflect antagonism at the higher level of selection, groups replicate at a rate that depends on the number of type $C$ individuals they contain. We take this rate to be $w_{G} \times[1+r(k / n)]$, where $k / n$ is the fraction of type $C$ individuals in the group and $r(x), x \in[0,1]$ is the selection coefficient at group level. The number of groups is maintained at $m$ by selecting a group uniformly at random to die whenever a group replicates. The two offspring of groups are assumed to be identical to their parent.

Two limits of large population are justified from the individual-based model in [20]. At least four different effects may contribute to the limiting dynamics. The selective effect at individual level is due to difference in growth rate between $I$ and $G$ individuals; the selective effect at group level to difference in the growth rate of groups (depending on the proportion of $G$ individuals). Consider the number of replacement events in a Moran model of a population made up of a constant number of identical individuals. In a limit of large populations as stated in the central limit theorem, this number shall increase with a linear rate with random fluctuations that may be well approximated by a Brownian Motion. Similar fluctuation effects generate variations in the proportion of trait carriers in a population. One can include such effects, that we call "random fluctuations", in the limiting large population process, both at individual and group levels.

Both limits in [20] include a term for each selective force, but only the second includes random fluctuations, both within the groups and between groups. In [26], another limit is considered, where random fluctuations are kept only inside the groups, together with selective forces. Although such limit can be mathematically justified, it is undoubtedly more realistic to keep also random fluctuations at group level. Yet, the analysis is notably much more technical with much less clear interpretation. In this more complete model, one type go almost surely extinct in finite time. Such event of "ultimate fixation" has a positive probability for each type. For large enough group populations, the limit obtained by neglecting these fluctuations provides an interesting view on the main features of the dynamics.

Let $X_{t}^{i}$ be the number of type $C$ individuals in group $i$ at time $t$. Then :

$$
\mu_{t}^{m ; n}:=\frac{1}{m} \sum_{i \leq m} \delta_{X_{t}^{i} / n}
$$

is the empirical measure at time $t$ of the proportion of type $C$ by group, with $m$ the number of groups and $n$ the number of individuals per group. Here, $\delta_{x}$ is the Dirac at $x$. The $X_{t}^{i}$ are divided by $n$ so that $\mu_{t}^{m ; n}$ is a probability measure on $E_{n}:=[0 ; 1 / n ; \ldots ; 1]$. For fixed $T>0,\left(\mu_{t}^{m ; n}\right)_{t \leq T} \in D\left([0 ; T] ; \mathcal{M}_{1}\left(E_{n}\right)\right)$, the set of càdlàg processes on $[0 ; T]$ taking values in $\mathcal{M}_{1}\left(E_{n}\right)$ (the set of probability measures on $E_{n}$ ). With the particle process described above, $\mu_{t}^{m ; n}$ has generator

$$
\left(\mathcal{L}^{m ; n} \psi\right)(v)=\sum_{i, j}\left(w_{I} R_{I}^{i, j}+w_{G} R_{G}^{i, j}\right)(v) \times\left(\psi\left[v+1 / m\left(\delta_{j / n}-\delta_{i / n}\right)\right]-\psi[v]\right)
$$

where $\psi \in C_{b}\left(\mathcal{M}_{1}([0 ; 1])\right)$ is a bounded continuous function, and $v \in \mathcal{M}_{1}\left(E_{n}\right) \subset \mathcal{M}_{1}([0 ; 1])$. 
The transition rates $\left(w_{I} R_{I}^{i, j}+w_{G} R_{G}^{i, j}\right)$ are given by

$$
R_{I}^{i, j}(v):= \begin{cases}m v(i / n) i(1-i / n)(1+s) & \text { if } j=i-1 ; i<n \\ m v(i / n) i(1-i / n) & \text { if } j=i+1 ; i>0 \\ 0 & \text { otherwise }\end{cases}
$$

$$
\text { and } \quad R_{G}^{i, j}(v):=m v(i / n) v(j / n)(1+r[j / n]) .
$$

$R_{I}^{i, j}$ and $R_{G}^{i, j}$ are the rates of respectively individual- and group-level reproductive events.

Theorem 2.1. Suppose that $w_{I} / n \rightarrow \omega_{I}, n s \rightarrow \sigma$ as $n, m \rightarrow \infty$, while $w_{G}$ and $\{r(x)\}_{x \in[0,1]}$ are kept constant. Suppose the particles in the process $\mu_{t}^{m ; n}$ are initially independently and identically distributed according to the measure $\mu_{0}^{m ; n}$, where $\mu_{0}^{m ; n} \rightarrow \mu_{0}$ as $m, n \rightarrow \infty$. Then, $\mu_{t}^{m ; n}$ converges weakly to $\mu_{t} \in D\left(\mathbb{R}_{+} ; \mathcal{M}_{1}([0 ; 1])\right)$, where $\mu_{t}$ is the unique solution with initial condition $\mu_{0}$ of the differential equation:

$$
\begin{gathered}
\partial_{t}\left\langle\mu_{t} \mid f\right\rangle=\left\langle\mu_{t} \mid \mathcal{L}_{W F} f\right\rangle+\left\langle\mu_{t} \mid r f\right\rangle-\left\langle\mu_{t} \mid f\right\rangle \times\left\langle\mu_{t} \mid r\right\rangle, \\
\text { where } \mathcal{L}_{W F} f(x)=-s x(1-x) \partial_{x} f(x)+\left(\sigma^{2} / 2\right) \cdot x(1-x) \partial_{x x}^{2} f(x) .
\end{gathered}
$$

\subsection{Definition as a conditional law}

We describe the solution of such equation through a Feynman-Kac penalization of the stochastic process with generator $\mathcal{L}_{W F}$. Let us define $X$ as the solution of the SDE :

$$
d X_{t}:=-s X_{t}\left(1-X_{t}\right) d t+\sigma \sqrt{X_{t}\left(1-X_{t}\right)} d B_{t}, \quad X_{0} \sim \mu_{0}
$$

The existence and uniqueness of such process can be found e.g. in chapter 5.3.1 of [12]. It is linked there with an individual-based model, namely the neutral 2-allele Wright-Fischer Markov Chains. We also consider the following Feynman-Kac penalization :

$$
Z_{t}:=\exp \int_{0}^{t} r\left(X_{s}\right) d s
$$

Note that $r$ is bounded so that for any $t>0, \mathbb{E}\left(Z_{t}\right) \in(0, \infty)$.

Proposition 2.2. With the above definitions, we characterize $\mu_{t}$ by the fact that for any $f \in \mathcal{C}_{b}^{2}$ :

$$
\left\langle\mu_{t} \mid f\right\rangle:=\mathbb{E}\left[f\left(X_{t}\right) Z_{t}\right] / \mathbb{E}\left[Z_{t}\right],
$$

I refer to [26] for the proof of the proposition, which is easily derived from the Ito formula.

We next give another view on this law $\mu_{t}$, that will simplify our notations and clarify our point. Since subtracting a constant to $r$ does not change the value of $\left\langle\mu_{t} \mid r f\right\rangle-\left\langle\mu_{t} \mid f\right\rangle\left\langle\mu_{t} \mid r\right\rangle$, we assume in the following that $r \leq 0$, so that we can consider it as a death rate. $Z_{t}$ can then be interpreted as the probability that the process has survived until time $t$, while confronted to a death rate of $r$, conditionally on $\left(X_{u}\right)_{u \geq 0}$. More formally, with $T_{\partial}$ an independent exponential r.v. with mean 1, we can define the extinction time as :

$$
\begin{gathered}
\tau_{\partial}:=\inf \left\{t \geq 0 ;-\ln \left(Z_{t}\right) \geq T_{\partial}\right\}, \quad \text { so that } \mathbb{P}\left(t<\tau_{\partial} \mid X_{u}, u>0\right)=\mathbb{P}\left(-\ln \left(Z_{t}\right)<T_{\partial} \mid X_{u}, u \leq t\right)=Z_{t} \\
\left\langle\mu_{t} \mid f\right\rangle=\mathbb{E}\left[f\left(X_{t}\right) ; t<\tau_{\partial}\right] / \mathbb{P}\left[t<\tau_{\partial}\right]=\mathbb{E}\left(f\left(X_{t}\right) \mid t<\tau_{\partial}\right) .
\end{gathered}
$$

Any equilibrium of $\mu_{t}$ is thus a quasi-stationary distribution (QSD) of $X$ under the death rate $r$. Applying recent results on QSD makes it possible to obtain the limiting behavior and the speed of convergence of $\mu_{t}$. 
Similarly, the hitting times of 0 and 1 are denoted $\tau_{0}$ and $\tau_{1}$. Since they are absorbing, it is natural to be interested in the law of the marginal with these extended extinction times:

$$
\tau_{0, \partial}:=\tau_{\partial} \wedge \tau_{0}, \quad \tau_{1, \partial}:=\tau_{\partial} \wedge \tau_{1}, \quad \tau_{0,1}:=\tau_{0} \wedge \tau_{1}, \quad \tau_{0,1, \partial}:=\tau_{\partial} \wedge \tau_{0} \wedge \tau_{1}
$$

Proposition 2.3. With the above definitions, we can also characterize $\mu_{t}$ by the fact that for any $f \in \mathcal{C}_{b}^{2}$ :

$$
\mu_{t}=x_{t}^{0} \delta_{0}+x_{t}^{1} \delta_{1}+x_{t}^{\xi} \xi_{t}
$$

$$
\begin{aligned}
\text { where } x_{t}^{\xi} & :=\frac{\mathbb{E}\left[Z_{t} ; t<\tau_{0,1}\right]}{\mathbb{E}\left[Z_{t}\right]}, \quad\left\langle\xi_{t} \mid f\right\rangle:=\frac{\mathbb{E}\left[f\left(X_{t}\right) Z_{t} ; t<\tau_{0,1}\right]}{\mathbb{E}\left[Z_{t} ; t<\tau_{0,1}\right]}=\mathbb{E}\left[f\left(X_{t}\right) \mid t<\tau_{0,1, \partial}\right], \\
x_{t}^{0} & :=\frac{\mathbb{E}\left[Z_{\tau_{0}} \exp \left[-r_{0}\left(t-\tau_{0}\right)\right] ; \tau_{0}<t\right]}{\mathbb{E}\left[Z_{t}\right]}, \quad x_{t}^{1}:=\frac{\mathbb{E}\left[Z_{\tau_{1}} \exp \left[-r_{1}\left(t-\tau_{1}\right)\right] ; \tau_{1}<t\right]}{\mathbb{E}\left[Z_{t}\right]}
\end{aligned}
$$

The proof is elementary and left to the reader.

Remarks : • It is not difficult to generalize the model to include frequency dependent effects of selection at individual level. We would then replace $s>0$ by some smooth function $(s(x))_{x \in[0,1]}$. Generalizations of $\sigma$ as a function are also not a mathematical issue. Yet a priori, it does not seem biologically justified.

- This Feynman-Kac penalization is analogous to the many-to-one formula described by [3] in the case of Markov processes associated to branching Galton-Watson Trees. Behind this description is the idea that there is a bias towards larger offspring in the genealogy of a typical individual in the population, favoring the individuals with a larger birth rate. Contrary to [3], where no interaction occurs between lineages, one cannot expect to represent the law of $\mu_{t}$ directly through another Markov Process, i.e. without penalization (note in (5) the quadratic term in $\mu_{t}$ ).

\subsection{QSDs and exponential convergence}

Note first that in any case, $\delta_{0}$ and $\delta_{1}$ are QSDs for the extinction time $\tau_{\partial}$, i.e. stable distributions for the dynamics given by (5). If the initial condition $\mu$ supported on $\{0,1\}$, the dynamics is immediately deduced from the death rates in 0 and 1.

For other initial conditions $\mu$ (not supported on $\{0,1\}$ ), we define the following semi-groups associated to our different extinctions :

$$
\mu A_{t}(d x):=\mathbb{P}_{\mu}\left(X_{t} \in d x \mid t<\tau_{\partial}\right), \quad \mu A_{t}^{01}(d x):=\mathbb{P}_{\mu}\left(X_{t} \in d x \mid t<\tau_{0,1, \partial}\right), \quad \mu A_{t}^{1}(d x):=\mathbb{P}_{\mu}\left(X_{t} \in d x \mid t<\tau_{1, \partial}\right)
$$

Proposition 2.4. There exists a unique $Q S D \alpha \in \mathcal{M}_{1}[(0,1)]$ and a survival capacity of $\eta$ associated to the extinction time $\tau_{0,1, \partial}$. With the associated extinction rate $\rho_{\alpha}$, it means :

$$
\forall t>0, \quad \mathbb{P}_{\alpha}\left(X_{t} \in d x ; t<\tau_{0,1, \partial}\right)=\exp \left[-\rho_{\alpha} t\right] \alpha(d x), \quad \forall x, \eta(x)=\exp \left[\rho_{\alpha} t\right] \mathbb{E}_{x}\left(\eta\left(X_{t}\right) ; t<\tau_{\partial}\right)
$$

Moreover, we have the following exponential convergences at rate $\zeta>0$ :

$$
\begin{gathered}
\exists C>0, \forall \mu \in \mathcal{M}_{1}[(0,1)], \quad\left\|\mu A_{t}^{01}-\alpha\right\|_{T V} \leq C \exp [-\zeta t] . \\
\exists C^{\prime}>0, \forall \mu \in \mathcal{M}_{1}[(0,1)], \quad\left|\exp \left[\rho_{\alpha} t\right] \mathbb{P}_{\mu}\left(t<\tau_{0,1, \partial}\right)-\langle\mu \mid \eta\rangle\right| \leq C^{\prime} \exp [-\zeta t] \\
\text { a fortiori } \eta(x):=\lim _{t \rightarrow \infty} \exp \left[\rho_{\alpha} t\right] \mathbb{P}_{x}\left(t<\tau_{0,1, \partial}\right) \quad \text { and }\|\eta \bullet\|:=\sup _{\{x \in(0,1), t>0\}} \exp \left[\rho_{\alpha} t\right] \mathbb{P}_{x}\left(t<\tau_{0,1, \partial}\right)<\infty
\end{gathered}
$$

Let $\rho_{0}=-r_{0}\left(\rho_{1}=-r_{1}\right)$ the extinction rate of $\delta_{0}$ (resp. $\left.\delta_{1}\right)$. We show in the following that the long-time behavior of the process with only the local extinction rate depends mainly on $\rho_{\alpha}, \rho_{0}$ and $\rho_{1}$. 
In the following convergences, we will often have uniform bounds for probability measures belonging for some $n \geq 1$ and $\xi>0$ to :

$$
\begin{aligned}
& \mathcal{M}_{n, \xi}^{0}:=\left\{\mu \in \mathcal{M}_{1}([0,1]) \mid \mu[1 / n, 1] \geq \xi\right\}, \bigcup_{n, \xi} \mathcal{M}_{n, \xi}^{0}=\mathcal{M}_{1}([0,1]) \backslash\left\{\delta_{0}\right\} \\
& \text { or in } \quad \mathcal{M}_{n, \xi}^{0,1}:=\left\{\mu \in \mathcal{M}_{1}([0,1]) \mid \mu[1 / n, 1-1 / n] \geq \xi\right\}, \quad(n \geq 3, \xi>0) \\
& \bigcup_{n, \xi} \mathcal{M}_{n, \xi}^{0,1}=\mathcal{M}_{1}([0,1]) \backslash\left\{u \delta_{0}+(1-u) \delta_{1} \mid u \in[0,1]\right\}
\end{aligned}
$$

2.2.1. Despite the fixation events, polymorphic groups maintain themselves in the population

Proposition 2.5. Assume that $\rho_{\alpha}<\rho_{0} \wedge \rho_{1}:=\rho$. Then, there is only one stable QSD $\alpha_{0,1}$, with convergence rate $\rho-\rho_{\alpha}$, i.e. :

$$
\forall n \geq 1, \forall \xi>0, \exists C_{n, \xi}>0, \quad \forall \mu \in \mathcal{M}_{n, \xi}^{0,1}, \quad\left\|\mu A_{t}-\alpha_{0,1}\right\|_{T V} \leq C_{n, \xi} \exp \left[-\left(\rho_{\alpha}-\rho\right) t\right],
$$

where $\alpha_{0,1}$ has extinction rate $\rho_{\alpha}$ and is given as $\alpha_{0,1}=y_{0} \delta_{0}+y_{1} \delta_{1}+y_{\alpha} \alpha$ with :

$$
\frac{y_{0}}{y_{\alpha}}=\frac{\rho_{\alpha} \times \mathbb{P}_{\alpha}\left(\tau_{0}=\tau_{0,1, \partial}\right)}{\left(\rho_{0}-\rho_{\alpha}\right)}, \quad \frac{y_{1}}{y_{\alpha}}=\frac{\rho_{\alpha} \times \mathbb{P}_{\alpha}\left(\tau_{1}=\tau_{0,1, \partial}\right)}{\left(\rho_{1}-\rho_{\alpha}\right)}
$$

and of course $y_{0}+y_{1}+y_{\alpha}=1$.

If $\rho_{1}<\rho_{0}$, for any initial condition $\mu_{0}=u \delta_{0}+(1-u) \delta_{1}$ with $u \in(0,1), \mu_{t}$ converges at rate $\rho_{0}-\rho_{1}$ to $\delta_{1}$.

If $\rho_{1}=\rho_{0}$, then any such distribution is a QSD with the extinction rate $\rho_{0}$.

Pure groups are continuously generated from polymorphic groups without any reversed transition. Yet, in this case, these polymorphic groups are sufficiently selected upon through their better survival to persist in the population. Pure groups are like remnants of these polymorphic groups : their proportion reaches a steady state where their faster decay compensate for the fixation rate from polymorphic groups. The stabilization of polymorphic profile induces the convergence of both this fixation rate and of the maintenance rate of polymorphic groups to $\rho_{\alpha}$.

In any case, polymorphism is maintained by any sufficiently large group selection favoring it, since :

Proposition 2.6. Given any $\sigma>0, s \geq 0$, and a bounded continuous function $r^{0}$ with its maximum only in the interior of $(0,1)$, there exists a critical value $R_{\vee}>0$ such that for any $R>R_{\vee}$ and considering the system with $r=R r^{0}$, we indeed have $\rho_{\alpha}<\rho_{0} \wedge \rho_{1}$.

Conversely, when group selection is too small, polymorphic groups cannot be maintained :

Proposition 2.7. Conversely, given any $\sigma>0, s \geq 0$, and a bounded measurable function $r^{0}$, there exists a critical value $R_{\wedge}>0$ such that for any $R<R_{\wedge}$ and considering the system with $r=R r^{0}$, it holds $\rho_{0} \wedge \rho_{1}<\rho_{\alpha}$.

Too strong neutral fluctuations also make the fixation of the groups hardly avoidable, so that :

Proposition 2.8. Given any $s>0$ and any bounded function $r, \lim _{\sigma \rightarrow \infty} \rho_{\alpha}(\sigma)=+\infty$.

Conditions for polymorphism to be maintained :

For this maintenance rate $\rho_{\alpha}$ to be higher than the decay of pure groups, it is clearly necessary that $r$ is maximal in $(0,1)$. Of course, it is not sufficient, because first of random fluctuations $(\sigma)$ and second because of selection effects inside each group. As stated in Proposition 2.6, too large $\sigma$ would induce a too large rate of fixation (to 0 or 1). Even strong effects of selection through $r$ would then be unable to make their reproduction large enough to compensate for this loss.

But on the other hand, when there are internal selective effects pushing towards type $I$ individuals, having only small random fluctuations limits the effectiveness of selection at group level. Indeed, all groups with similar initial condition evolve too closely for such effects of selection to really distinguish between them : they are essentially driven by the flow of the ODE : $\partial_{t} x_{t}:=-s x_{t}\left(1-x_{t}\right)$. Even if perturbations are amplified by this selection towards the opposite direction, strong deviations would be much too costly. 
2.2.2. Fixation on either side is the most stable case and the type $C$ is favored by group selection

Proposition 2.9. Assume that $\rho_{1}<\rho_{0}<\rho_{\alpha}$. Then, $\delta_{1}$ is the only stable QSD, with convergence rate $\rho_{0}-\rho_{1}:$

$$
\forall n \geq 1, \forall \xi>0, \exists C_{n, \xi}>0, \quad \forall \mu \in \mathcal{M}_{n, \xi}^{0}, \quad\left\|\mu A_{t}-\delta_{1}\right\|_{T V} \leq C_{n, \xi} \exp \left[-\left(\rho_{0}-\rho_{1}\right) t\right]
$$

We also have an additional level of convergence :

Proposition 2.10. Assume that $\rho_{0}<\rho_{\alpha}$. Then, there exists $C>0$ s.t. :

$$
\forall \mu \in \mathcal{M}_{1}([0,1]) \backslash\left\{\delta_{1}\right\}, \quad\left\|\mu A_{t}^{1}-\delta_{0}\right\|_{T V} \leq C \exp \left[-\left(\rho_{\alpha}-\rho_{0}\right) t\right]
$$

Both results are asymptotic and might not reflect exactly the dynamics on a short time-scale. Yet, their justification gives us some insight into what can happen.

When polymorphism gets quickly negligible :

If $\sigma$ is large, except for initial conditions very close to 0 , a non-negligible proportion of pure type $C$ groups quickly emerges and dominates the distribution. For initial conditions very close to 0, the emergence time of these pure $C$ groups mainly depends on the proportion of groups able to quickly escape such vicinity of 0 . Soon, the growth in the proportion of pure $C$ groups is essentially due to the difference in growth rate between these groups and the rest of the population, meaning that, at this time, the fixation of polymorphic groups plays a negligible role. Finally, one observes the competition between the two types of pure groups, with the initially rare $G$ groups outnumbering the first dominant $I$.

When trajectories are drifted with little fluctuations :

As explained in the previous subsection, the flow of the equation $\partial_{t} x_{t}:=-s x_{t}\left(1-x_{t}\right)$ dominates the dynamics as long as $\mu_{t}$ stays localized. If $\sigma$ is rather small, the profile $\mu_{t}$ is essentially given by the integration of the growth rate along the trajectories of the flow. Notably, consider for simplicity the case where the initial condition is supported on $[0,1-2 \epsilon]$. The pure flow brings the group from a proportion $1-\epsilon$ to $\epsilon$ in a deterministic time $t_{\epsilon}$. If $\sigma$ is small enough, then with a probability close to 1 the process is upper-bounded by this deterministic flow starting from $1-2 \epsilon$. It implies that $\mu_{t_{\epsilon}}$ is mainly concentrated in $[0,2 \epsilon]$. If the proportion of pure $G$ groups is still negligible at this time, we shall observe a decay in the proportion of polymorphic groups larger than an exponential rate of $\rho_{\alpha}-\rho_{0}$. It is a priori unclear that the rate $\rho_{\alpha}-\rho_{0}$ is actually observed, since the QSD $\alpha$ might be localized around 1 and very difficult to reach for initial conditions with many less cooperative groups. One may expect some stabilization to occur, where $\mu_{t}$ restricted to $(0,1)$ gets close to some $\tilde{\alpha}$. This distribution $\tilde{\alpha}$ is presumably supported mainly close to 0 , with a much larger extinction rate $\rho_{\tilde{\alpha}}$ than $\rho_{\alpha}$ (and $\left.\rho_{0}\right)$. One shall have the right intuition by considering $\tilde{\alpha}\left(\operatorname{resp} . \rho_{\tilde{\alpha}}\right)$ instead of $\alpha\left(\operatorname{resp} . \rho_{\alpha}\right)$ in the reasoning of Propositions 2.9 and 2.10. This view is supported by first hints of simulations (not detailed in the article).

As stated in Proposition 2.9, pure $G$ groups shall prevail even in that case. A rate $\rho_{1}-\rho_{0}$ of decay of remaining groups is very likely to be seen, but possibly after a domination by type I groups for a large period of time. The duration of this domination depends actually much on the initial distribution, and particularly on its tail near 1 . Indeed, transitions to 1 is especially costly in term of its probability of occurrence.

Compensate the flow of invasion by I individuals :

Section 3 provides an evaluation of the strength of this group selection needed to compensate the flow in the limit of vanishing $\sigma$. In this large deviation regime, the process seems to evolve for most of the time according to a modification of the initial flow. Yet, it is not as simple : for instance, one might observe the abrupt emergence of a type which was so far negligible, whose growth rate is much higher than the previously dominant type.

This is exactly what shall presumably happen in this model in the case $r_{1}<r_{0}$. Undoubtedly, the initial proportion of $G$ groups or the neutral fixation for initially almost pure $G$ groups concerns a very tiny proportion of ancestors. Yet, the ancestors initially drifted towards larger proportion of $I$ individuals soon loose any $G$ 
individual (with very few exceptions). So there is a point in time at which we can no longer neglect the more prolific descendants the former exceptional ancestor groups will have. This shall certainly correspond to the time at which $G$ individuals eventually dominate. Are the ones that finally dominate necessarily pure groups ? Due to the potential long-term persistence of the process in the vicinity of 1 when the random fluctuations are very small, the behavior of $\rho_{\alpha}$ as $\sigma$ tends to 0 is quite unclear. So it might happen that some almost pure $G$ groups actually dominate.

\section{Selection upon the initial condition :}

Still, the selection between different initial conditions may be effective if those are sufficiently apart. It may postpone for some significant time the trend towards 0 . Yet, for the polymorphism to persist for long, a very specific form of the law of the initial condition is required. This has been specified in [20] in the limit where the random fluctuations are neglected. Such long-term persistence is effectively possible because the flow is vanishing in the vicinity of 1 . The authors consider only functions $r$ that are linear in the proportion of type $G$ individuals. But it should generalize to a much more general cases, provided $r$ is larger near 1 than near 0 .

Note however that in such regime, the groups dominating at a given time are essentially not the ancestors of those dominating at a much larger time. The descendants of the former have been transported by the flow towards 0 , where this sub-population has decayed faster. On the other hand, the ancestors of the latter must have stayed for long in a very tiny and specific region very close to 1, and are thus very few (while the former dominate). Indeed, going backward through the ancestry lines means essentially following the flow backwards. This backward flow goes quickly towards 1 , which it approaches at exponential speed. With initial conditions irregular in the vicinity of 1 , we thus might observe a surprising sequence of vanishing and reemerging polymorphism between periods of domination by $I$ individuals.

Is this observable in the individual-based model?

Any transition involving a reasonable amount of groups is expected to be indeed observed. This can be estimated through the number of ancestors from the initial population upon which such transitions shall rely. The fluctuations shall vanish with the number of them. Provided the neutral fluctuations in the births and deaths of groups are small, the approximation should be qualitatively valid with approximately 20 ancestries.

It means also that too exceptional transitions are very unlikely to be observed. For instance, the escape from a too close vicinity of 0 happens with a too small probability. The most likely is to observe the complete fixation. For the fixation of pure $C$ groups to be observed, the most probable is then to have one group escaping the vicinity of 0 , reaching the other boundary and generating a sufficiently large family there for the extinction to become negligible. Only after such exceptional realization becomes the fixation of pure $C$ groups likely to occur. Given Theorem 2.1, larger population sizes makes the event more likely to occur. Yet, in order for one group to behave in a way so different from the typical one, it might be required that the population size is at a largely unrealistic level. Similarly, when $\sigma$ is so small that transitions towards 1 become negligible, and for an initial condition with a light tail (towards 1), the fixation of pure $C$ groups happens after a very exceptional behavior.

This mathematical complexity is presumably not so relevant in terms of the biology. As soon as the initial condition has a sufficiently light tail in this vicinity of 1 , one mainly observe a massive proportion of the groups fixing as pure $I$ types and becoming dominant for a very long time.

\subsubsection{Polymorphic groups are more stable than type I groups but less than pure type $C$ groups}

This case is also treated in [26]. The result is a combination of the ones in the two previous subsections. The dynamics for the domination by pure $G$ groups relies on similar principles as in Proposition 2.9. The main difference is that the intermediate convergence is stated towards a polymorphic QSD rather than pure $I$ groups. This polymorphic QSD $\alpha_{1}$ is described as in Proposition 2.5 when one subtracts pure $C$ groups before the renormalization. The asymptotic rate of convergence towards the Dirac at pure $G$ groups is deduced from this intermediate convergence result : $\rho_{1}-\rho_{\alpha}$ (smaller than $\left.\rho_{1}-\rho_{0}\right)$. 
Again, for the approximated IBM, one may be faced to the same limitations regarding the origin of the first pure $G$ groups as in Proposition 2.9. In practice, the convergence towards the polymorphic QSD might also not actually reflect the main dynamics of convergence. It might happen that the "emergence" of pure type 1 groups can actually be almost concomitant to the emergence of $\alpha^{1}$. Looking at simulations for small values of $\sigma$, an alternative metastable regime around 0 may dominate for a significant time the marginal law restricted to $(0,1)$. Comparing with the case of initial condition close to 1 , this distribution $\tilde{\alpha}$ is very different from the actual QSD $\alpha$, with very separate supports. Even if $\rho_{\alpha}<\rho_{0}$, it would not be surprising that for the alternative distribution $\rho_{\tilde{\alpha}}>\rho_{0}$. For a large range of initial conditions, even if $\rho_{\alpha}<\rho_{0}$, the initially observed dynamics is rather the one described in Propositions 2.9 and 2.10 by the interplay between 1,0 and $\tilde{\alpha}$ where $\rho_{1}<\rho_{0}<\rho_{\tilde{\alpha}}$. The first step of the dynamics is thus obviously a convergence to 0 with a rate expected to stabilize towards $\rho_{\tilde{\alpha}}-\rho_{0}$.

\subsubsection{Validation by some simulations?}

In order to evaluate the exceptionality of transitions towards 1, one can propose the following simulation experiment, which is a work in progress. Let us consider some parameters for which most of the marginals tend towards 0 , with $\sigma$ sufficiently small. To observe the upheaval of the group population by type $G$ groups, the marginals should be encoded rather not by the masses at the different grid points, but by the logarithms of these quantities. The case of the absorbing states is treated separately from the marginal restricted to $(0,1)$. For a better accuracy, it may be useful to refine the grid in the vicinities of 0 and 1 .

We do observe the mass towards 1 increasing up to the point of exceeding the mass towards 0 . Yet, it is then unclear at which concentration the regeneration of these cooperative groups exceeds the effect of having more and more exceptional transitions leading there. Since the quickest transitions are expected to bring less mass towards 0 , our idea is to truncate densities to prevent the most exceptional transitions. So at each simulation step, we suppress from the marginal the mass on states than contain less than the threshold. By varying the threshold, we should have a better view on the number of groups required to observe the transition from 0 to 1 in individual-based models.

If the dynamics is almost unchanged after the truncation, we could conclude that the cost of the transitions that mainly contribute to this upheaval is smaller than the threshold. If the upheaval arises later on, it would mean that less costly transitions could have been sufficient to make type $G$ emerge. Yet, their contribution becomes negligible when compared to quicker transitions. Finally, if the marginal becomes supported on some interval that does not approach 1 , it means that any transition towards 1 would be at least as costly as the threshold.

\subsection{Conclusion of the section}

Generally, the interplay between different traits happens in the time-scale at which their carriers can be differentiated. Yet, we have seen in this example that the trade-off between different kinds of advantages can be particularly tricky. The a priori neutral genetic drift might happen to be strongly coupled to the efficiency of some components of selection. In a broader view, we can see this model as an illustration that selective effects might be strongly dependent upon details of the local ecological dynamics, and not only upon the average behavior. If the local subdivision constitutes a sufficiently stable entity with the ability to reproduce itself, natural selection may act. Its strength depends on the level of variability between those entities, as if they were individuals.

Crucial requirements for the presented confrontation are the independence of the groups bewteen successive splittings and a strong heritability for the groups when they split (the two descendants are assumed to be very similar to the "parent"). Even small interactions between these communities (notably migration between groups) is known to greatly disrupt the stability of cooperative strategies (cf. for instance [27]). So we clearly do not claim that such simplified effect is prevalent, because such lack of interaction between the local dynamics is not so common. 
Also, the selection at the group level might rely on exceptional transitions of the process $X$. Although this exceptionality can be compensated in the long run by a larger asymptotic growth rate, two aspects should be remembered : first, it might be much too unlikely for actual populations that some groups experience those rare transitions, so that the mathematical model could be misleading; second, it takes a very long time for their descendants to invade. It thus raises the question concerning real life whether no other event happens to disturb any of the sub-populations before the emergence of cooperative groups.

Finally, this competition model provides a fruitful insight in an evolutionary perspective (that is the subject of the following Section 4). A main quantity of interest is notably the probability that the descendants of a single mutant individual with trait $y$ invade and replace the whole population of "residents" with trait $x$. This probability is usually compared to the one for the invasion by mutants identical to the residents (the neutral case). In this model, for small values of $\sigma$, as long as the mutant trait has a strong deleterious effect, either at the individual- or at the group-level, the invasion is much more difficult than neutral : for the invasion of cooperatives, the random fluctuations inside the groups have to lead the process away from the very stable cheater quasi-equilibrium; while, for the invasion of cheaters, the genetic drift between groups has to disrupt the also very stable cooperative quasi-equilibrium. Such large deviations are known to be generally especially costly. It means that natural selection of such traits should be much more constraint.

\section{LARGE DEVIATION ESTIMATE FOR THE ADAPTATION BY MUTATIONS OF WEAK EFFECT}

In this section, I present the work of Champagnat and Henry on some effects of Dirac concentration in non-local models of adaptation with several resources [10]. The focus is on the trajectories of evolution, with the selection of favorable mutations.

Interestingly, the authors of [10] show a mathematical similarity between two limiting behaviors : a first one where the trait variations are very small; and the second where the mutation rate is very small (as compared to the selective effects). Notably, the first case can be associated to a limit where mutations of very small effects accumulate. The main requirement for the proofs is that natural selection is very strong in the timescale where one observes the trait dispersion under neutrality.

Again, the population size is assumed to be sufficiently large to include the whole range of the stochastic variations of the trait. The resulting purely deterministic model shall provide a valid approximation to the dynamics of trait proportions in this population. This relation to the trait proportions can be retrieved from the convergence result deduced from the time-scale separation.

\subsection{The continuous-space limiting behavior}

One considers the family $\left(u^{\epsilon}\right)_{\epsilon}$ of deterministic solutions to the parabolic SDE :

$$
\begin{aligned}
& \partial_{t} u^{\epsilon}(t, x)=\frac{\epsilon}{2} \Delta u^{\epsilon}(t, x)+\frac{R\left(x, \psi_{t}^{\epsilon}\right)}{\epsilon} u^{\epsilon}(t, x), \quad x \in \mathbb{R}^{d}, t \geq 0, \\
& -\epsilon \log \left(u^{\epsilon}(0, x)\right)=h_{\epsilon}(x)
\end{aligned}
$$

where the competition effect $\psi_{t}^{\epsilon}=\left(\psi_{t}^{i, \epsilon}\right)_{i \leq r}$ is defined, for $r$ resources, with a competition kernel $\Psi_{i}: \mathbb{R}^{d} \rightarrow \mathbb{R}_{+}$ for resource $i$ by :

$$
\psi_{t}^{i, \epsilon}:=\int_{\mathbb{R}^{d}} \Psi^{i}(y) u^{\epsilon}(t, y) d y .
$$

In this model, the traits $x \in \mathbb{R}^{d}$ characterize the ability to exploit the different resources. Considering mutation effects through some heat kernel corresponds to the case where lots of mutations with very small effects occur and are dispersed throughout the population. In asexual populations, we rather expect selective mutations to invade and fix one after the other. Such a model is thus rather justified for sexual populations, where many recombination events occur and many different alleles with small selective effects may coexist. This is the principles of the so-called infinitesimal model for which we refer notably to [5]. For instance, it is known that 
many Human traits are affected by many different loci in the genome. Two individuals with almost the same phenotype have possibly very different alleles in these loci and the recombination of alleles along the lineages creates variability. The heat kernel seems then quite relevant to characterize the variability of response for such a trait. It would be of interest to prove that the dynamics of $u^{\epsilon}$, i.e. as a solution to 11 , can be justified as a limiting description of individual-based models as in Theorem 2.1.

The following assumptions are required in [10] in order to justify a limit to $\varphi_{\epsilon}:=-\epsilon \log u^{\epsilon}$ through a variational representation.

1. Assumptions on $\Psi_{i}$ : For any $1 \leq i \leq r, \Psi_{i} \in W^{2, \infty}\left(\mathbb{R}^{d}\right)$.

Moreover, there exist $0<\Psi_{\min }<\Psi_{\max }$ such that $\forall 1 \leq i \leq r,, \forall x \in \mathbb{R}^{d}, \quad \Psi_{\min } \leq \Psi_{i}(x) \leq \Psi_{\max }$.

2. Assumptions on $R$ : (a) $R$ is continuous on $\mathbb{R}^{d} \times \mathbb{R}^{r}$.

(b) There exists $A>0$ such that

$$
\forall 1 \leq i \leq r, \forall x \in \mathbb{R}^{d}, \forall\left(v_{\ell}\right) \in \mathbb{R}^{r}, \quad-A \leq \partial_{v_{i}} R\left(x,\left(v_{\ell}\right)_{\ell \leq r}\right) \leq-A^{-1}
$$

(c) There exist two positive constants $0<v_{\min }<v_{\max } \operatorname{such}$ that $\min _{x \in \mathbb{R}^{d}} R(x, v)>0$ as soon as $\|v\|_{1}<v_{\min }$, and $\max _{x \in \mathbb{R}^{d}} R(x, v)<0$ as soon as $\|v\|_{1}>v_{\max }$, where $\|v\|_{1}=\sum_{\ell \leq r}\left|v_{\ell}\right|$.

(d) Let $H$ denotes the annulus $B\left(x, 2 v_{\max }\right) \backslash B\left(x, v_{\min } / 2\right)$ (for the $\|\cdot\|_{1}$ norm). Then $\sup _{v \in H}\|R(\Delta, v)\|_{W^{2, \infty}}<\infty$.

3. Assumptions on $h_{\epsilon}$ : (a) $h_{\epsilon}$ is Lipschitz-continuous on $\mathbb{R}^{d}$, uniformly with respect to $\epsilon>0$.

(b) $h_{\epsilon}$ converges uniformly as $\epsilon$ tends to 0 to a function $h$.

(c) For all $\epsilon>0$ and all $1 \leq i \leq d, v_{\min } \leq \int_{\mathbb{R}^{d}} \Psi_{i}(x) \exp \left(-h_{\epsilon}(x) / \epsilon\right) d x \leq v_{\max }$.

In particular, $u_{\epsilon}(0, x)$ is bounded in $L^{1}\left(\mathbb{R}^{d}\right)$.

Remark : Such competition may at first sight appear as a global competition where every trait is competing against each other for the same resources. The first assumption done in [10] requires indeed for the $\Psi_{i}$ to be lower-bounded by a strictly positive constant. However, in a limit where $r$ is large and $\Psi_{i}$ is very concentrated, the model may become a valid approximation of a local competition (in the trait space). Thus, one may hope to extend the model naturally to such a framework.

\subsection{Results for the continuous case}

I gather here the main results presented in [10], where I have changed some notations for clarity, notably $\psi_{t}$ and $\varphi$ :

Theorem 3.1. (Feynman-Kac representation of the solution of (11))

Let $u^{\varepsilon}$ be the unique weak solution of (11), then, under the assumptions given in [10] :

$$
\forall(t, x) \in \mathbb{R}_{+} \times \mathbb{R}^{d}, \quad u^{\varepsilon}(t, x)=\mathbb{E}_{x}\left[\exp \left(\frac{-h_{\varepsilon}\left(X_{t}^{\varepsilon}\right)}{\varepsilon}+\frac{1}{\varepsilon} \int_{0}^{t} R\left(X_{t}^{\varepsilon}, \psi_{t-s}^{\varepsilon}\right) d s\right)\right]
$$

where for all $x \in \mathbb{R}^{d}, \mathbb{E}_{x}$ is the expectation associated to the probability measure $\mathbb{P}_{x}$, under which $X_{0}^{\varepsilon}=x$ almost surely and the process $B_{t}=\left(X_{t}^{\varepsilon}-x\right) / \sqrt{\varepsilon}$ is a standard Brownian motion in $\mathbb{R}^{d}$.

Remark : This representation of the solution through a penalization of a stochastic process is in practice of the same kind as the one presented for the empirical law $\mu_{t}$ in Section 2. There is only a little difference in the fact that $X_{t}$ describes now the process when we look at the lineage backward in time. A similar convergence result should thus justify the interpretation of $u^{\epsilon}(t, x) d x$ as the limit of some individual-based measure-valued processes $\left(\nu_{t}^{\epsilon, K}\right)$. In particular, $(1 / \epsilon) \cdot R\left(x, \psi_{t}^{\epsilon, K}\right)$ should be the additional growth rate of individuals with trait $x$, where for $i \leq r, \quad \psi_{t}^{i, \epsilon, K}:=\left\langle\nu_{t}^{\epsilon, K} \mid \Psi^{i}\right\rangle$. With fixed $\epsilon$, this is a priori a specific case of the McKean-Vlasov 
equations mentioned in the conclusion of Section 1, where the law of the process itself acts on the individual dynamics (again, I refer to [25]).

Lemma 3.2. The function $I_{t}^{R, \varepsilon}: C([0, t]) \rightarrow \mathbb{R}$ defined by $I_{t}^{R, \varepsilon}(y)=\int_{0}^{t} R\left(y_{s}, \psi_{s}^{\varepsilon}\right)$ ds is Lipschitz continuous on $C([0, t])$ endowed with the $L^{\infty}$-norm. The Lipschitz constant is uniform with respect to $\varepsilon$ for $\varepsilon$ small enough. Moreover, there exists a kernel $\mathcal{M}$ on $\mathbb{R}_{+} \times \mathcal{B}\left(\mathbb{R}^{k}\right)$ such that, along a subsequence $\left(\varepsilon_{k}\right)_{k \geq 1}$ converging to 0 :

$$
\forall y \in C([0, t]) \quad I_{t}^{R}(y):=\lim _{k \rightarrow \infty} I_{t}^{R, \varepsilon_{k}}(y)=\int_{0}^{t} \int_{\mathbb{R}^{k}} R\left(y_{s}, \psi\right) \mathcal{M}_{s}(d \psi) d s .
$$

By a kernel $\mathcal{M}$, we specify here a function from $\mathbb{R}_{+} \times \mathcal{B}\left(\mathbb{R}^{k}\right)$ into $\mathbb{R}_{+}$such that, for all $s \in \mathbb{R}_{+}, \mathcal{M}_{s}$ is a measure on $\mathcal{B}\left(\mathbb{R}^{k}\right)$ and, for all $A \in \mathcal{B}\left(\mathbb{R}^{k}\right)$, the function $s \rightarrow \mathcal{M}_{s}(A)$ is measurable.

Theorem 3.3. For all $(t, x)$ in $\mathbb{R}_{+} \times \mathbb{R}^{d}$,

$$
\varphi(t, x):=\lim _{k \rightarrow \infty} \varepsilon_{k} \log u^{\varepsilon_{k}}(t, x)=\sup _{y \in \mathcal{G}_{t, x}}\left\{-h\left(y_{0}\right)+I_{t}^{R}(y)-I_{t}^{L}(y)\right\}
$$

where the convergence holds uniformly on compact sets and the limit $\varphi(t, x)$ is Lipschitz w.r.t. $(t, x) \in \mathbb{R}_{+} \times \mathbb{R}^{d}$ while $I_{t}^{L}(y)= \begin{cases}\frac{1}{2} \int_{0}^{t}\left\|y^{\prime}(s)\right\|^{2} d s & \text { if } y \text { is absolutely continuous } \\ +\infty & \text { otherwise. }\end{cases}$

$\mathcal{G}_{t, x}$ denotes the set of continuous functions from $[0, t]$ to $\mathbb{R}^{d}$ such that $y_{t}=x$, and $I_{t}^{R}$ and $\left(\varepsilon_{k}\right)_{k \geq 1}$ are associated as in Lemma 3.2.

Remarks : • In this optimization, $y$ defines the main ancestral line of the individuals dominant with trait $x$ at time $t$. Namely, the Large Deviation theory ensures that as $\epsilon \rightarrow 0$, their ancestral lines are very concentrated on such specific and deterministic histories.

- Given a lineage described by $y, I^{R}(y)$ encodes an average effect of selection due to the perceived growth rate. As one can infer from (13) when $\epsilon$ is very small, the density around such a path is approximately amplified by the exponential of this quantity divided by $\epsilon$. Such large amplification is the core of the Large Deviation theory, where the stochastic behavior is concentrated close to specific paths.

- The dependency of the competition kernel $\mathcal{M}_{s}$ on $\varphi$ is implicit and unclear in the limiting model. The level of competition depends on the global composition in traits of the population, while $\varphi$ only indicates which traits are non-negligible (the $x$ for which $\varphi(x)=0$ ). Detailed information on the density is lost in the limit $\epsilon \rightarrow 0$, and that is why subsequences $\epsilon_{k}$ are considered. For the same reason, the uniqueness of solutions to such equations is not easy to establish (when it holds).

Again in [10], the authors finally relate this variational limit to the solution of the Hamilton-Jacobi equation :

Theorem 3.4. Under some assumptions on the behavior of $R$ and $h_{\epsilon}, \psi^{\varepsilon_{k}}$ converges in $L_{\text {loc }}^{1}\left(\mathbb{R}_{+}\right)$along the subsequence $\varepsilon_{k}$ of Lemma 3.2 to a nondecreasing limit $\bar{\psi}$, the kernel $\mathcal{M}$ satisfies

$$
\forall s \geq 0, \quad \mathcal{M}_{s}(d \psi)=\delta_{\bar{\psi}_{s}}(d \psi)
$$

and the limit $\varphi$ of Theorem 3.3 solves in the viscosity sense :

$$
\begin{cases}\partial_{t} \varphi(t, x)=R\left(x, \bar{\psi}_{t}\right)+\frac{1}{2}|\nabla \varphi(t, x)|, & \forall t \geq 0, x \in \mathbb{R}^{d}, \\ \max _{x \in \mathbb{R}^{d}} \varphi(t, x)=0, & \forall t \geq 0 .\end{cases}
$$

Behavior of the solution to Hamilton-Jacobi equation.

From Theorem 3.3, one deduces the following approximation for small $\epsilon: u^{\epsilon}(t, x) \approx C(t, x) \exp [\varphi(t, x) / \epsilon]$. We thus expect to observe a concentration of the individual traits on typically a unique value or a few wellseparated values. In the limiting model, these values may be driven by a continuous displacement in the direction of natural selection. They may also split, which would in a sense describe a model of speciation. It is in fact the original purpose of such Hamilton-Jacobi analysis in [14] to extend the model of Adaptive Dynamics (cf. 
Subsection 4.5). The reverse can happen too, with two subpopulations merging into a single one : the traits are concentrated around a moving value specific to each subpopulation until these two values coalesce. Finally, there could also be some jumps, that is a brutal change of the trait composition. A wide variety of events are thus observable with this simplified model.

Discussion on the involved time-scales.

At a given $\epsilon>0$, looking at equation (11), we can infer from the growth rate that the stabilization of the densities occurs in a time-scale of order $\epsilon$, as compared to the time-scale at which the traits evolve. The variance of the trait evolves on the contrary at a much larger time scale of order $\epsilon^{-1}$, as in the case of neutral traits. Of course, the detailed description of the demography as presented in Section 1 above and in [18] should occur in the short time-scale of order $\epsilon$. In such a large population, the life-expectancy of any particular individual is on an even shorter time-scale. depending on the ratio between the birth and the death rates. The closer it is to one, the shorter this time-scale. A separation of time-scale with the evolutionary trajectory can still be justified for the competition between different effects of selection. But as we have seen in Section 2, this competition might last for very long times.

In practice, the description given by equation (11) is not so easily related to the macroscopic observation of the response to selection, because the neutral variability is difficult to scale (the factor $\epsilon$ before the Laplacian). We can reasonably assume that we know the density $u^{e}(t, x)=u^{\epsilon}(t, x)$ (as long as it is not too negligible) and the fitness effect $R_{t}^{e}(x)=(1 / \epsilon) \cdot R\left(x, \psi_{t}\right)$. For at least positive values, it can be inferred in some laboratory experiment by artificially introducing individuals with trait $x$ in a much larger sample of the population at time $t$ and see how they grow. One could then estimate the value $\epsilon$ for which $\varphi^{\epsilon}(t, x)=\epsilon \log u^{e}(t, x)$ seems reasonably close to the solution $\varphi$ of the Hamilton-Jacobi equation :

$$
\partial_{t} \varphi(t, x)=\epsilon R_{t}^{e}(x)+\frac{1}{2}|\nabla \varphi(t, x)|,
$$

or the associated control problem.

Note that a Gaussian distribution for $u^{e}$ corresponds to $\varphi(t, x) \sim-\left(x-x_{t}\right)^{2} / \sigma_{t}^{2}$, that is the Second order Taylor expansion around $x_{t}$, with $x_{t}$ the optimal trait and $\epsilon \sigma_{t}^{2}$ the variance of the distribution. Moreover, if $\varphi$ is a solution to equation (14), then for $\lambda>0, \hat{\varphi}(x, t):=\varphi(\lambda t, \lambda x)$ is a solution to (14) with $\epsilon$ replaced by $\lambda \epsilon$. Since we expect the dependency in $\sigma_{0}$ on $\sigma_{t}$ to vanish very quickly, the identification of $\epsilon$ from $\sigma_{t}$ should not be a too difficult option. Yet, as mentioned in the next paragraph, the speed of response to selection might be driven by an exceptional proportion of the population at time $t$. An estimate such as $\sigma_{t}$ that summarizes rather the core of the distribution $u^{e}(t, x) d x$ might thus not be so relevant.

Connection with individual-based models.

To justify the connection with individual-based models, concentration effects may appear favorable. Yet, as we can see from Theorem 3.3, the adaptation of the population is driven by exceptional profiles of stochastic variations. The stochastic techniques of Large Deviations is then crucial to obtain the limiting behavior. Notably, it means that selection is mainly driven by individuals at the front. This optimization provides a very interesting insight on the history of the genealogies that led to the traits observed generally in the population at time $t$. Notably, we can observe cases where the traits at time $s \leq t$ for the ancestors of the dominants at time $t$ may not be typical at all as compared to the dominant traits at time $s$. This of course raises the issue of the biological relevance of such transitions. We face similar limitations as in previous Section 2 for the relevance of events involving very small population sizes. Still, it is much easier to relate the above description to some individual-based model than from the more classical description only relying on Hamilton-Jacobi equations (cf. the conclusion of the section). 


\subsection{Results for the discrete case}

This analysis of Feynman-Kac operators and Large Deviation principles can be adapted to a discrete space. Simplifying assumptions are then more easily obtained and makes it possible to have a clearer view on the implications of this model.

In [10], the authors consider the following system of ordinary differential equations :

$$
\left\{\begin{array}{l}
\partial_{t} u^{\varepsilon}(t, k)=\sum_{j \in E \backslash\{k\}} \exp \left(\frac{-\mathfrak{T}(k, j)}{\epsilon}\right)\left(u^{\varepsilon}(t, j)-u^{\varepsilon}(t, k)\right)+\frac{1}{\varepsilon} u^{\varepsilon}(t, k) R\left(k, \psi_{t}^{\varepsilon}\right), \quad \forall t \in[0, T], \quad \forall k \in E \\
u^{\varepsilon}(0, k)=\exp \left(-\frac{h(k)}{\varepsilon}\right), \quad \psi_{t}^{i, \varepsilon}=\sum_{k \in E} \Psi^{i}(k) u^{\varepsilon}(t, k), \quad \forall i \leq r
\end{array}\right.
$$

In such a model, mutations from state $j$ to state $i$ happen at rate $\exp \left(-\epsilon^{-1} \mathfrak{T}(k, j)\right)$. As mentioned in the introduction of [10], this is not the classical form of the solution for the system of ODE describing population densities. The former can however be obtained from the latter by a slight adjustment in the definition of $R$ (vanishing as $\epsilon \rightarrow 0$ ) and is more practical for the following analysis. The growth rate of the individuals in state $k$ is (nearly) $\epsilon^{-1} R(k, \psi)$, where $\psi=\left(\psi^{i}\right)$ specifies the amount of available resources.

First, the solution $u^{\varepsilon}$ of the system is described in [10] by using an integral representation similar to (13). Let $\left(X_{s}^{\varepsilon}, s \in[0, T]\right)$ be the Markov process in $E$ with infinitesimal generator :

$$
L^{\varepsilon} f(k)=\sum_{j \in E} \exp \left[\frac{-\mathfrak{T}(k, j)}{\varepsilon}\right](f(j)-f(k))
$$

i.e. the continuous-time Markov process whose jump rate from state $i \in E$ to $j \neq i$ is $\exp (-\mathfrak{T}(i, j) / \varepsilon)$.

Proposition 3.5. (Integral representation)

For any positive real number $t$ and any element $i$ of $E$, we have

$$
u^{\varepsilon}(t, i)=\mathbb{E}_{i}\left[\exp \left(\frac{-h\left(X_{t}^{\varepsilon}\right)}{\varepsilon}+\frac{1}{\varepsilon} \int_{0}^{t} R\left(X_{s}^{\varepsilon}, \psi_{t-s}^{\varepsilon}\right) d s\right)\right]
$$

The interpretation is very similar to the one of Theorem 3.1.

Proposition 3.6. (Weak LDP) $\left(X^{\varepsilon}\right)_{\varepsilon \geq 0}$ satisfies a weak $L D P$ with rate function

$$
I_{T}:\left\{\begin{array}{l}
\mathrm{D}([0, T], E) \rightarrow \mathbb{R} \\
y \mapsto \sum_{\ell=1}^{N_{y}} \mathfrak{T}\left(y_{t_{\ell}^{y}}, y_{t_{\ell}^{y}}\right)
\end{array}\right.
$$

where $\mathrm{D}([0, T], E)$ is the space of càdlàg functions from $[0, T]$ to $E, N_{y}$ is the number of jumps of $y$ and $\left(t_{\ell}^{y}\right)_{1 \leq \ell \leq N_{y}}$ the increasing sequence of jump times of $y$.

We shall use the notation $\quad I_{T}(y):=\sum_{0<s \leq T} \mathfrak{T}\left(y_{s-}, y_{s}\right) \quad$ with the implicit convention that $\mathfrak{T}(i, i)=0$ for all $i \in E$.

Theorem 3.7. Lemma 3.2 is also satisfied in the discrete case and for all $(t, i)$ in $(0,+\infty) \times E$, with the associated subsequence $\left(\varepsilon_{k}\right)$ :

$$
\begin{aligned}
& \varphi(t, i):=\lim _{k \rightarrow \infty} \varepsilon_{k} \log u^{\varepsilon_{k}}(t, i) \\
& =\sup _{y \in \mathrm{D}([0, t], E) \text { s.t. } y_{0}=i}\left\{-h\left(y_{t}\right)+\int_{0}^{t} \int_{\mathbb{R}^{r}} R\left(y_{s}, \psi\right) \mathcal{M}_{t-s}(d \psi) d s-\sum_{0<s \leq t} \mathfrak{T}\left(y_{s-}, y_{s}\right)\right\} .
\end{aligned}
$$

As in the expression in Theorem 3.3, one shall remark that the optimal $y$ in this expression describes the transition of states for the lineage of a typical individual at time $t$. This evolution is backward in time, as can be also seen in the term $R\left(y_{s}, \psi\right) \mathcal{M}_{t-s}(d \psi)$, where $\mathcal{M}_{t-s}(d \psi)$ provides the law of $\psi_{t-s}$.

Theorem 3.8. For all subsequence $\left(\varepsilon_{k}\right)_{k \geq 1}$ as in Theorem 3.7, the limit $\varphi(t, i)$ of $\varepsilon \log u^{\varepsilon}(t, i)$ is Lipschitz with respect to the time variable $t$ on $(0,+\infty)$. In addition, if $h(i) \leq h(j)+\mathfrak{T}(i, j)$ for all $i \neq j$, the function $\varphi$ is Lipschitz on $\mathbb{R}_{+}$. 
We see that all the previous results in continuous space have an equivalent for discrete space.

In the following, we focus on a specific case where the uniqueness can be obtained and the whole dynamics described with much more accuracy. The result presented in [10] relies on some assumption on the stability of the dynamics restricted to any subset of $E$, which is called Assumption $(H)$. I present next the main intuition behind this assumption and refer to [10] for the exact formulation.

By this Assumption $(H)$, the authors notably ensure that, for any subset $A$ containing $k$ different types, there exists a unique strongly attractive equilibrium. Since the focus is on the dynamical system, the estimation of the dynamics is valid as soon as the $k$ types are initially in non-negligible proportion. Yet, some of the components of the steady-state may be 0, meaning an exponential decay of the mass of those. Assumption $(H)$ also ensures that any other (unstable) equilibrium is quickly escaped. The dominant traits at time $t($ for which $\varphi(t,)=0$. are then proved to stay piecewise constant, while the emergence of favorable competitors is easily computed. Once such a competitor has emerged, it disrupts the equilibrium of traits, and assumption $(H)$ enables us to predict the issue of the equilibrium that follows.

Given any set $A$ of present types, the equilibrium is given by $\left(u_{A, j}^{*}\right)_{j \in A}$. Thus, the competition exerted on the $i$-th resource by this eco-system is $\Psi^{i}(A):=\sum_{j=1}^{r} \Psi^{i}(j) \cdot u_{A, j}^{*}$.

Remark : To satisfy assumption $(H)$, these steady states need to satisfy a condition of compatibility regarding their vanishing components. By restricting the dynamics on some subset $B$ of $A$ that contains all the non-vanishing components of the steady state associated with $A$, the steady state for $B$ is necessarily the restriction on $B$ of the steady state for $A$.

Proposition 3.9. Assume that $(H)$ is satisfied. Let $\left(\varepsilon_{k}\right)_{k \geq 1}$ be as in Theorem 3.7. For any $t \geq 0$, there exists $\rho_{t}>0$ such that, for all $s \in\left(t, t+\rho_{t}\right], \psi_{s}^{\varepsilon}$ converges to $\psi_{s}=\psi_{t}:=\Psi(\{\varphi(t, \cdot)=0\})$, where the convergence is uniform in all compact subsets of $\left(t, t+\rho_{t}\right]$ and where we define for any $A \subset E: \quad F(A)=$ $\left(\sum_{j=1}^{r} \eta_{i}(j) u_{A, j}^{*}\right)_{1 \leq i \leq r}$.

In particular, the weak limit $\mathcal{M}_{s}$ of $\delta_{\psi_{s}}$ obtained in Lemma 3.2 satisfies $\mathcal{M}_{s}=\delta_{\psi_{t}}$, for almost all $s \in\left(t, t+\rho_{t}\right)$ and the function $t \mapsto \psi_{t}$ is right-continuous.

As in the previous case, we shall observe a concentration effect. It means here that there are brutal transitions between one demographic equilibrium and the next one generated by the first invasion by a mutant sub-population.

Proposition 3.10. Assume that $(H)$ is satisfied. Any limit $\varphi$ of $\varepsilon_{k} \log u^{\varepsilon_{k}}$ along a subsequence as in Theorem 3.7 satisfies for all $i \in E$ and for all $t \geq 0: \varphi(0, i)=-h(i)$

$$
\varphi(t, i)=\sup _{y(0)=i}\left\{-h(y(t))+\int_{0}^{t} R\left(y(u), \psi_{t-u}\right) d u-I_{t}(y)\right\}
$$

and its dynamic programming version

$$
\varphi(t, i)=\sup _{y(s)=i}\left\{\varphi(s, y(t))+\int_{0}^{t} R\left(y(u), \psi_{t+s-u}\right) d u-I_{s, t}(y)\right\} .
$$

In addition, the problem (15) admits a unique solution s.t. $t \mapsto \psi_{t}:=F(\{\varphi(t, \cdot)=0\})$ is right-continuous. In particular, the full sequence $\left(\varepsilon \log u^{\varepsilon}\right)_{\varepsilon>0}$ converges to this unique solution when $\varepsilon \rightarrow 0$.

Theorem 3.11. Under Hypothesis $(H)$ and assuming that $h(i) \leq h(j)+\mathfrak{T}(i, j)$ for all $i \neq j$, the problem

$$
\left\{\begin{array}{l}
\partial_{t} \varphi(t, i)=\sup \left\{R\left(j, \psi_{t}\right) \mid j \in E \text { s.t. } \varphi(t, j)-\mathfrak{T}(j, i)=\varphi(t, i)\right\} \\
\forall i \in E, \quad \varphi(0, i)=h(i) \quad \text { ( with the convention } \mathfrak{T}(i, i)=0)
\end{array}\right.
$$


admits a unique solution such that $t \mapsto \psi_{t}=F(\{\varphi(t, \cdot)=0\})$ is right-continuous and it is the unique solution to the variational problem (15).

The system follows a succession of equilibria (with possibly different types that equilibrate), with the population headcount of mal-adapted types vanishing linearly in their logarithm, while this headcount logarithm increases for the rare adapted ones. It goes on until one adapted type reaches the threshold for a non-negligible frequency. Then occurs a kind of "catastrophe", where the whole equilibrium is immediately renewed (in the time-scale of evolution). Given the similarity between the description in this discrete case and the continuous case presented in Subsection 3.2, one can infer that similar "catastrophes" might happen even in the continuous case. This is less expected, since we see meanwhile a dynamics for the dominant trait, but it cannot be excluded a priori that a brutal change of the traits happen.

Without this assumption of a unique stable equilibrium, it would not be clear what happens to the population during such a "catastrophe" event. Namely, the only information provided by $\varphi(., t)$ is the knowledge of the non-negligible types, for which $\varphi(x, t)=0$, with a priori no means to infer otherwise the headcounts at equilibrium. Notably, different issues or an unstable behavior could lead to different competition effects, thus different dynamics after the "catastrophe".

The main restriction for this model is that the tails of the distribution are not too much involved. Indeed, when there is some barrier, where the growth rate is very low, the trajectory that gets selected upon through the optimization procedure in Theorem 3.3 might not be realistic. It might well be inferred from this optimization that the ancestors of the population at time $t$ shall represent at time $s \leq t$ a proportion less than $10^{-9}$ of the entire population at time $s$. Of course, it would only be reasonable for populations with a size quite larger than $10^{9}$ ! And all the more since families with small population size have a high risk of going extinct. Considering the actual population size considered, such optimization procedure could be an efficient way to truncate too rare such transitions as we have proposed for the analysis in Section 2. Namely, for a threshold $-\varphi_{\vee}$, we would like to define some $\widetilde{\varphi}(t, x)$ as the supremum over the same quantity as in Theorem 3.3 under the additional condition on $y \in \mathcal{G}_{t, x}$ that :

$$
\forall s \leq t, \quad-h\left(y_{0}\right)+I_{s}^{R}(y)-I_{s}(y)>-\varphi_{\vee}, \quad \text { where } I_{s}^{R}(y):=\int_{0}^{s} \int_{\mathbb{R}^{k}} R\left(y_{u}, \psi\right) \widetilde{\mathcal{M}}_{u}(d \psi) d u
$$

Naturally, we want to impose $\widetilde{\varphi}(t, x)=-\infty$ if no function $y \in \mathcal{G}_{t, x}$ is able to satisfy this condition. By this way, we forbid transitions that rely on too exceptional ancestors to be treated in such a deterministic way. While $\widetilde{\mathcal{M}}_{u}$ shall represent the availability of resources at time $u$, it shall actually depend on $\widetilde{\varphi}$, as in the case without truncation. This makes the analysis a priori much trickier. Yet, the transitions we forbid are rare so there is a lag between the time at which they occur and the time at which they have an effect on $\widetilde{\mathcal{M}}$. The estimation of $\widetilde{\mathcal{M}}$ shall thus not be more difficult than the one of $\mathcal{M}$ in the case without truncation.

Alternative strategies have been proposed in [24] and [23] with more regularities than the proposed truncation, yet not much more biological justification. To prevent such exceptional densities, the authors use a singular term for the growth rate, which gets very negative when the density of the state is too small.

To be even more realistic, one should treat such events of crossing barriers as punctual events occurring at a very low rate. In fact, large deviation approaches may be well suited to estimate these rates. One should only remark that the growth rate on the other side of the barrier is not responsible for any increase on the rate at which such transitions occur. It only increases the probability that such exceptional crossing event leads actually to an invasion. And that is why such events are much more exceptional than with the asymptotic model given by $\varphi$, where the growth rate immediately and regularly increases the log-density on this other side. The punctual transitions that we are to describe in the next section is thus in fact well suited to deal also with such rare crossing events. 


\section{Mutations as the Limiting FaCtor}

Although this section is clearly referring to the talk given by Tran, the presentation he made was not dedicated to a specific paper. It was rather a general introduction to the framework of IBM at use to prove classical scaling limits of adaptive dynamics. The focus was mostly on models where the traits are highly conserved along the lineages until exceptional events of mutation in a new-born. Relying on two articles of my choice that are in the spirit of Tran's talk, I intend to offer a broad perspective on the limiting descriptions of IBM. Given the progression of the whole paper, I favored in my choice (besides the commitment of the speaker) the coupling of several time-scales over the intrinsic complexity in the asymptotic dynamics.

This last Section 4 addresses the longest time-scales, where the limiting factor for evolution is the emergence of mutations. By its emergence, we mean not only that some individuals carry such mutations at time $t$ but rather that it has been stabilized at a non-negligible frequency in the population. For simplicity, many authors assume that selective traits cannot coexist in the same population, and that the population size is very stable at a value depending on this dominant trait. Namely, demographic fluctuations that has been crucial in Section 2 are neglected, and a fortiori the individual variability detailed in Section 1 is only seen through an average effect. In such a deterministic approximation, the fixation or the decay of an invasive trait is generally described, as soon as the proportion of invaders is not negligible anymore, by Lotka-Volterra equations. For elementary models of interactions (notably not frequency-dependent), it is then easy to impose conditions ensuring that the fixation of one of the alleles is the only stable equilibrium of the dynamical system.

Given the assumption that such ecological transitions happen in a much shorter time-scale than the time between occurrences of new mutations (at first in a unique individual), such conditions, called "invasion implies fixation", ensures that the population stays almost always monomorphic in the evolutionary time-scale. This means in particular that the invasion of new mutations can be treated as punctual events that refreshes the equilibrium. In this view, this behavior is similar to the evolution of traits given for the discrete space in Section 3 (cf. the concluding remarks for more details on this link). Yet, these punctual fixation events happen at unpredictable times, corresponding to the occurrence of a successful mutation.

Note that this shall hold true even if coexistence of traits are allowed in the model. Yet, the rate at which an invasion occurs and its issue might depend on any variations of the frequencies (like oscillation of frequencies) and of the population size, which makes the analysis tricky. Of course, one could also generalize these models to include competition for resources as in Section 3. Yet, the specificity of these more complicated models can only be seen when several traits can coexist. If one wishes to include events of speciation, this is however a very reasonable way to justify it (specialization of two sub-populations).

For strong selection effects, one can prove negligible the time-interval between the arrival of the first mutant and the invasion of the population by its descendants (at least reaching a non-negligible frequency). Namely, a favorable mutant has a larger expectancy of offspring than individuals of resident type and shall thus generate a family that either dies out quite early or thrive at an exponential rate. Contrary to the model of discrete trait evolution described in Subsection 3.3, the time-scale for the occurrence of mutations is chosen here in such a way that this growth period is not a limiting factor. By "successful mutation", we mean the occurrence of the mutation in a first carrier followed by a successful invasion by its descendants of the resident population. By assuming that mutation events are sufficiently rare, we infer that successful mutations happen nearly independently of the time waiting for it (where the monomorphic population stays the same) and of unsuccessful invasions. This explains why the time-interval between two successive events of invasion is given by an exponential law without memory.

Such a process with piecewise constant population equilibria, including a dominant trait, has been originally introduced as the Trait Substitution Sequence in [22] and more formally related to individual-based models in [8]. A large family of extensions has emerged, notably to include coexistence of traits (cf. [11], [1]), or more complicated interactions, for instance with horizontal transfer [6] or aging [21]. Since they deal with two additional time-scales, we rather focus on [7], where events of invasion affect the dynamics of some marker, 
and [2], which demonstrates that adaptive dynamics can indeed be an accurate approximation of some individualbased models.

\subsection{A model combining a marker dynamics with the evolution of traits}

The next four subsections present the results of Billiard, Ferrière, Méléard and Tran on the stochastic dynamics of neutral markers coupled to the one of adaptive traits [7]. The authors consider an asexual population driven by births and deaths where each individual is characterized by hereditary types: a phenotypic trait under selection and a neutral marker. This analysis is motivated by research on the prevalence of selection between various species or clades that could be based on the observed variability of neutral markers.

The trait and marker spaces $\mathcal{X}$ and $\mathcal{U}$ are assumed to be compact subsets of $\mathbb{R}$. The type of individual $i$ is thus a pair $\left(x_{i}, u_{i}\right), x_{i} \in \mathcal{X}$ being the trait value and $u_{i} \in \mathcal{U}$ its neutral marker. The individual-based microscopic model from which we start is a stochastic birth and death process with density-dependence whose demographic parameters are functions of the trait under selection and are independent of the marker. We assume that the population size scales with an integer parameter $K$ tending to infinity so that individuals are weighted with $\frac{1}{K}$ to observe a non-trivial limit of the empirical measure. The state of the population at time $t \geq 0$, rescaled by $K$, is described by the point measure :

$$
\nu_{t}^{K}=\frac{1}{K} \sum_{i=1}^{N_{t}^{K}} \delta_{\left(x_{i}, u_{i}\right)}=X_{t}^{K}(d x) \pi_{t}^{K}(x, d u), \quad \text { where } X_{t}^{K}=\frac{1}{K} \sum_{i=1}^{N_{t}^{K}} \delta_{x_{i}} \text { and } \pi_{t}^{K}(x, d u)=\frac{\sum_{i=1}^{N_{t}^{K}} \mathbf{1}_{x_{i}=x} \delta_{u_{i}}}{\sum_{i=1}^{N_{t}^{K}} \mathbf{1}_{x_{i}=x}}
$$

are respectively the trait marginal and the marker distribution for a given trait value $x$. Here, $\delta_{(x, u)}, \delta_{x}$ are respectively the Dirac measure at $(x, u)$ and $x$.

With the following definitions, the authors ensure that the mutations happen at different time scales for the trait and for the marker, both longer than the individuals lifetime scale. Thus, the limiting behavior results from the interplay of these three time scales: births and deaths, trait mutations and marker mutations. To justify such a separation of time scales for the mutations, the proof relies strongly on the fact that the population size remains around the equilibrium of some dominant trait(s) when a mutation on the trait occurs. Although the authors are able to include fluctuations of the parameter $u$, it is only possible because $u$ has no effect on the probability that the mutation succeeds to invade the population.

\subsection{The individual-based model}

Definition 4.1. - An individual with trait $x$ and marker $u$ reproduces with birth rate given by $0 \leq b(x) \leq \bar{b}$, the function $b$ being continuous and $\bar{b}$ a positive real.

- Reproduction produces a single offspring which usually inherits the trait and marker of its ancestor except when a mutation occurs. Mutations on trait and marker occur independently with probabilities $p_{K}$ and $q_{K}$ respectively. Mutations are rare and the marker mutates much more often than the trait. We assume that

$$
q_{K}=p_{K} r_{K}, \text { with } p_{K}=\frac{1}{K^{2}}, q_{K} \underset{K \longrightarrow \infty}{\rightarrow} 0, r_{K} \underset{K \longrightarrow \infty}{\longrightarrow}+\infty .
$$

- When a trait mutation occurs, the new trait of the descendant is $x+k \in \mathcal{X}$ with $k$ chosen according to the probability measure $m(x, k) d k$.

- When a marker mutation occurs, the new marker of the descendant is $u+h \in \mathcal{U}$ with $h$ chosen according to the probability measure $G_{K}(u, d h)$. For any $u \in \mathcal{U}, G_{K}(u,$.$) is approximated as follows when K$ tends to infinity:

$$
\lim _{K \rightarrow+\infty} \sup _{u \in \mathcal{U}}\left|\frac{r_{K}}{K} \int_{\mathcal{U}}(\phi(u+h)-\phi(u)) G_{K}(u, d h)-A \phi\right|=0,
$$

where $(A, \mathcal{D}(A))$ is the generator of a Feller semigroup and $\phi \in \mathcal{D}(A) \subseteq C_{b}(\mathcal{U}, \mathbb{R})$, the set of continuous bounded real functions on $\mathcal{U}$. 
- An individual with trait $x$ and marker $u$ dies with intrinsic death rate $0 \leq d(x) \leq \bar{d}$, the function $d$ being continuous and $\bar{d}$ a positive real. Moreover, the individual experiences competition the effect of which is an additional death rate

$$
\eta(x) . C * \nu_{t}^{K}(x)=\frac{\eta(x)}{K} \sum_{i=1}^{N_{t}^{K}} C\left(x-x_{i}\right) .
$$

The quantity $C\left(x-x_{i}\right)$ describes the competition pressure exerted by an individual with trait $x_{i}$ on an individual with trait $x$. We assume that the functions $C$ and $\eta$ are continuous and that there exists $\underline{\eta}>0$ such that

$$
\forall x, y \in \mathcal{X}, \eta(x) C(x-y) \geq \underline{\eta}>0 .(2.6)
$$

A classical choice of competition function $C$ is $C \equiv 1$ which is called "mean field case" or "logistic case". In that case the competition death rate is $\eta(x) N_{t}^{K} / K$.

As mentioned in the beginning of the Section 4, the authors work under the simplifying assumption that follows, ensuring that the population remains monomorphic between two events of invasion by a new trait.

Assumption "Invasion implies fixation" For all $x \in \mathcal{X}$ and for almost every $y \in \mathcal{X}:$

$$
\text { either } \frac{b(y)-d(y)}{\eta(y) C(y-x)}<\frac{b(x)-d(x)}{\eta(x) C(0)}, \quad \text { or } \frac{b(y)-d(y)}{\eta(y) C(y-x)}>\frac{b(x)-d(x)}{\eta(x) C(0)} \text { and } \frac{b(x)-d(x)}{\eta(x) C(x-y)}<\frac{b(y)-d(y)}{\eta(y) C(0)} \text {. }
$$

Remark : $\quad \hat{\mathrm{n}}_{x}:=\frac{b(x)-d(x)}{\eta(x) C(0)}$ is the equilibrium of the dynamical system that a large population size of individuals with trait $x$ approximate.

Moreover, in the case of logistic populations with a constant $C$, this assumption is satisfied as soon as $x \rightarrow \hat{\mathrm{n}}_{x}$ is strictly monotonous.

\subsection{Flemming-Viot process and marker evolution}

The dynamics of the marker is first defined for a constant trait $x$ via a Flemming-Viot process as defined below. This process generalizes to a potential infinity of markers the measure-valued process describing two markers : $F_{t}(d v)=X_{t} \delta_{0}(d v)+\left(1-X_{t}\right) \delta_{1}(d v)$ with $X$ the neutral Wright-Fisher diffusion defined in Section 2, cf. (7) with $s=0$ and remarks below.

In the sequel, we denote by $\mathcal{P}(\mathcal{U})$ and $\mathcal{P}(\mathcal{X} \times \mathcal{U})$ the probability measure spaces respectively on $\mathcal{U}$ and on $\mathcal{X} \times \mathcal{U}$, while $\langle\nu \mid \phi\rangle$ denotes the integral of the measurable function $\phi$ against the measure $\nu$. Also, $\mathcal{M}_{F}(\mathcal{X} \times \mathcal{U})$ is the set of finite measures on $\mathcal{X} \times \mathcal{U}$.

Definition 4.2. Given $x \in \mathcal{X}$ and $u \in \mathcal{U}$, the Fleming-Viot process $\left(F_{t}^{u}(x, t \geq 0)\right.$ indexed by $x$, started at time 0 with initial condition $\delta_{u}$ and associated with the mutation operator $A$ is the $\mathcal{P}(\mathcal{U})$-valued process whose law is characterized as the unique solution of the following martingale problem. For any $\phi \in \mathcal{D}(A)$,

$$
M_{t}^{x}(\phi)=\left\langle F_{t}^{u}(x, .) \mid \phi\right\rangle-\phi(u)-b(x) \int_{0}^{t}\left\langle F_{s}^{u}(x, .) \mid A \phi\right\rangle d s
$$

is a continuous square integrable martingale with quadratic variation process

$$
\left\langle M^{x}(\phi)\right\rangle_{t}=\frac{2 b(x)}{\hat{n}_{x}} \int_{0}^{t}\left(\left\langle F_{s}^{u}(x, .) \mid \phi^{2}\right\rangle-\left\langle F_{s}^{u}(x, .) \mid \phi\right\rangle^{2}\right) d s .
$$

Remark : The model presented in Section 2 provides simple illustrations for such kind of processes. For the solution $X$ of (7), define :

$$
F_{t}(d v):=X_{t} \delta_{0}(d v)+\left(1-X_{t}\right) \delta_{1}(d v) .
$$


Then, with $A \equiv 0$ (the only transitions for the traits are between 0 and 1 ), $S$ a continuous function such that $S(0)=s$ (the parameter of selection at individual level) and $S(1)=0$, for any $\varphi$ measurable :

$$
\begin{aligned}
& M_{t}(\phi):=\left\langle F_{t} \mid \phi\right\rangle-\left\langle F_{0} \mid \phi\right\rangle-\left(\left\langle F_{t} \mid S \times \phi\right\rangle-\left\langle F_{t} \mid S\right\rangle \times\left\langle F_{t} \mid \phi\right\rangle\right) \\
& =[\phi(0)-\phi(1)]\left(X_{t}-x-\int_{0}^{t} s X_{r}\left(1-X_{r}\right) d r\right)=[\phi(0)-\phi(1)] \sigma \int_{0}^{t} \sqrt{X_{r}\left(1-X_{r}\right)} d B_{r},
\end{aligned}
$$

where we used Ito's formula, is clearly a square-integrable martingale with quadratic variation :

$$
\langle M(\phi)\rangle_{t}=[\phi(0)-\phi(1)]^{2} \sigma^{2} \int_{0}^{t} X_{r}\left(1-X_{r}\right) d r=\sigma^{2} \int_{0}^{t}\left(\left\langle F_{r} \mid \phi^{2}\right\rangle-\left\langle F_{r} \mid \phi\right\rangle^{2}\right) d r .
$$

Note that $s$ is here a selective effect on the distribution that is not present in (16) because the marker is neutral.

Moreover, recalling the equation that described the state $\mu_{t}$ of the population of groups in Section 2 , one can relate it to equation (16) with $b(x) A=\mathcal{L}_{W F}$ and the martingale $M(\phi)$ being identically zero (i.e. with zero quadratic variations). In [20], the authors in fact derive another description of the population of groups in the limit of large population sizes (intra-groups and inter-groups). This limit is also described as such FlemmingViot process, with a non-zero martingale because one does no longer neglect the non-selective birth and death events of groups. Again, its quadratic variations satisfies : $d\langle M(\phi)\rangle_{t} \propto \int_{0}^{t}\left(\left\langle F_{u} \mid \phi^{2}\right\rangle-\left\langle F_{u} \mid \phi\right\rangle^{2}\right) d u$. Of course, there is still the additional term involving $r$ in the finite variation part (16). I refer for instance to [16] and [13] for a detailed presentation of Flemming-Viot processes.

\subsection{Convergence to the Substitution Flemming-Viot Process}

We can now state the main theorem that describes the slow-fast dynamics of adaptive traits and neutral markers at the (trait) evolutionary time scale.

Theorem 4.3. We work under Definition 4.1 and the assumption that "invasion implies fixation". Consider the initial condition $\nu_{0}^{K}(d y, d v)=n_{0}^{K} \delta_{\left(x_{0}, u_{0}\right)}(d y, d v)$ with $\lim _{K \rightarrow \infty} n_{0}^{K}=\hat{\mathrm{n}}_{x_{0}}$ and $\sup _{K \in \mathbb{N}^{*}} \mathbb{E}\left(\left(n_{0}^{K}\right)^{3}\right)<+\infty$. Then, the population process $\left(\nu_{K t}^{K}, t \geq 0\right)$ converges in law to the $\mathcal{M}_{F}(\mathcal{X} \times \mathcal{U})$-valued process $\left(V_{t}(d y, d v), t \geq 0\right)$.

To define this Markov process, with initial condition $\hat{n}_{x_{0}} \delta_{x_{0}} \delta_{u_{0}}$, we only need to describe it until the first jump of the trait, which is given by an exponential law. Namely, the trait jumps from $x_{0}$ to $x_{0}+k$ with rate:

$$
b\left(x_{0}\right) \hat{\mathrm{n}}_{x_{0}} \frac{\left[f\left(x_{0}+k ; x_{0}\right)\right]_{+}}{b\left(x_{0}+k\right)} m\left(x_{0}, k\right) d k .
$$

Then, given that this first jump occurs at time $T$, the law of the new marker is given by:

$$
U \sim F_{T}^{u_{0}}\left(x_{0}, d u\right), \text { so that } V_{T}(d y, d v)=\hat{n}_{x_{0}+k} \delta_{x_{0}+k} \delta_{U} .
$$

Then, for any $t<T$, we have:

$$
V_{t}(d y, d v)=\hat{\mathrm{n}}_{x_{0}} \delta_{x_{0}}(d y) F_{t}^{u_{0}}\left(x_{0}, d v\right),
$$

and the process is defined recursively like this before the next jumps.

The convergence holds in the sense of finite dimensional distributions on $\mathcal{M}_{F}(\mathcal{X} \times \mathcal{U})$. In addition, the convergence also holds in the sense of occupation measures, i.e. the measure $\nu_{K T}^{K}(d y ; d v) d t$ on $\mathcal{X} \times \mathcal{U} \times[0 ; T]$ converges weakly to the measure $V_{t}(d y ; d v) d t$ for any $T>0$.

This process is called by the authors the Substitution Fleming-Viot Process (SFVP). It generalizes the Trait Substitution Process (TSP) introduced in [22] and also obtained as a limit of individual-based model in [8]. The TSP is in fact the marginal of the SFVP on the trait space. The jump rate of the TSP can be easily interpreted. 
The mutation rate of any resident is given by $b\left(x_{0}\right) m\left(x_{0}, k\right) d k$. The number of such residents at equilibrium is nearly $\hat{n}_{x_{0}} K$. While in competition with the resident population, the survival of the lineage of the mutant depends mainly on the period where the associated sub-population is too small to disrupt the resident population. From classical results of Branching process, it survives with probability $\left[f\left(x_{0}+k ; x_{0}\right)\right]_{+} / b\left(x_{0}+k\right)$. Note that only beneficial mutations pass through, so that they shall invade quickly after reaching a non-negligible proportion in the population. The product provides the rate of occurrence of such successful mutation in the whole population, that gets divided by $K$ in the new time-scale.

Given the recent and impressive progress in sequencing and comparing genetic data between species, one has partly access to the marker dynamics. The selective dynamics is however much more difficult to follow, since one would have to evaluate mutation effects and the advantage they bring in the past eco-systems. It would thus be of high interest to be able to infer strong selective effects from the dynamics of the marker.

At each sweep, a very specific marker is selected. This effect is referred to as a genetic hitchhiking. It shall be chosen according to the law $F_{T}^{u_{0}}\left(x_{0}, d u\right)$. We may expect that numerous selective sweeps should increase the variability of the markers. Yet, without assuming any effect on the marker dynamics from the selective component, it is not very clear that doing these steps more frequently shall increase the variance in the selected marker (after a comparable time span).

Nonetheless, such hitchhiking events usually leave a mark, at least for sexual reproduction species. Because of frequent recombination events, hitchhiking effect is mostly effective for markers closely linked to the selected allele. The diversity of variants become small that one gets closer in the genome to the selected allele. A well-known example is given by the selection for genes that favor the digestion of milk. As one can imagine, the analysis is then much more demanding than the convergence to the SFVP.

\subsection{The last time-scale of adaptive-dynamics}

As the last time-scale presented in the current paper, Adaptive Dynamics is probably the one that gives to natural selection the most predictable effect. Let us follow Baar, Bovier and Champagnat [2] : the connection of Adaptive dynamics with the individual-based model is demonstrated through a single step of convergence (as long as no singularity is reached). In this context, the canonical equation of adaptive dynamics (CEAD) states that the population of interest can be considered monomorphic, and its trait $x_{t}$ follows an ordinary differential equation of first order. Namely, the speed of the trait involves the mutation rate, the population size at equilibrium, some derivative of the fitness of invasion, and the squared effect of mutations in the direction of invasion. Remark that this last term is not the mean effect of mutations in this direction because the more effective is a mutation, the more likely it is to fix. In order to obtain such deterministic behavior, we again need the assumptions for the TSS, i.e. rare mutations as compared to the ecological time-scale, with negligible fluctuations around the size equilibrium and invasion implying fixation (cf. previous Subsection). Moreover, the CEAD relies on the assumption that mutations have infinitesimal effects, so that the trait evolves continuously by the accumulation of large number of such mutations. The connection of the TSS to the CEAD involves a coupled rescaling of time and of fitness effects, which is rather natural. Yet, for the actual connection with the individual-based model, it introduces the major difficulty that any favorable mutation step shall be quite insignificant and yet shall replace effectively the dominant trait. In the same idea, the dominant population shall prevent deleterious yet almost neutral mutations to invade and filter favorable mutation with an invasion success still proportional to the mutation effect.

Nonetheless, the authors of [2] actually manage to demonstrate a regime of convergence to the CEAD, where these issues are rigorously controlled. Their individual-based model is quite close to the previous one, except that there is no marker anymore and that the possible mutation steps are assumed to be on some discrete and finite grid (whose mesh size goes to 0), preventing large mutations. I thus use the same notations as previously (rather than the one of [2]) and refer to Subsection 4.2. The main difference is also a scaling parameter for the mutation effect : 
When a trait mutation occurs (in a population with trait $x$ ), the new trait of the descendant is $x+\sigma_{K} k \in \mathcal{X}$

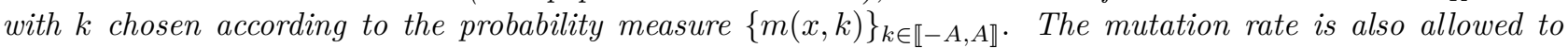
depend on $x$, and is thus given by $q_{K} M(x)$.

Besides the other assumptions we have made in Subsection 4.2, there are additional issues of regularity for the birth rate $b$, the death rate $d$, the mutation rate $M$, the sensibility to competition $\eta$ and the competition kernel $C$, for which assumptions are required. It is also assumed that $b(x)>d(x)$ and that $C(x, x)$ is uniformly upper-bounded for any $x \in \mathcal{X}$.

There is a last assumption to ensure the absence of singularity, based on the invasion fitness of a mutant $y$ in a resident population $x$ :

$$
f(y, x):=b(y)-d(y)-\eta(y) C(y, x) \hat{n}_{x} .
$$

It indicates the mean growth rate of a mutant population with trait $y$ as long as it is still negligible as compared to the resident population with trait $x$. As stated in the convergence to the TSS, the invasion probability of a mutant population initiated by a single individual with trait $y$ tends to $f(y, x)_{+} / b(y)$ (for large population size). Here, $f_{+}$is the positive part of $f$, meaning that deleterious mutations cannot invade.

Assumption 3 : For all $x \in \mathcal{X}, \quad \partial_{1} f(x, x) \neq 0$.

Assumption 3 implies that either $\forall x \in \mathcal{X}: \partial_{1} f(x, x)>0$ or $\forall x \in \mathcal{X}: \partial_{1} f(x, x)<0$. Therefore, coexistence of two traits is not possible. Without loss of generality, we can assume that, $\forall x \in \mathcal{X}, \partial_{1} f(x, x)>0$.

Theorem 4.4. Assume that Assumptions 1 and 3 hold and that there exists a small $\alpha>0$ such that

$$
\begin{aligned}
K^{-1 / 2+\alpha} & \ll \sigma_{K} \ll 1 \\
\text { and } \quad \exp \left(-K^{\alpha}\right) & \ll q_{K} \ll \frac{\sigma_{K}^{1+\alpha}}{K \ln K}, \quad \text { as } K \rightarrow \infty .
\end{aligned}
$$

Fix $x_{0} \in \mathcal{X}$ and let $\left(N_{0}^{K}\right)_{K \geq 0}$ be a sequence of $\mathbb{N}$-valued random variables such that $N_{0}^{K} / K$ converges in law, as $K \rightarrow \infty$, to the positive constant $\hat{\mathrm{n}}\left(x_{0}\right)$ and is bounded in $\mathrm{L}^{p}$, for some $p>1$.

For each $K \geq 0$, let $\nu_{t}^{K}$ be the process generated by $\mathcal{L}^{K}$ with monomorphic initial state $\left(N_{0}^{K} / K\right) . \delta_{\left\{x_{0}\right\}}$. Then, for all $T>0$, the sequence of rescaled processes, $\left(\nu_{t /\left(K q_{K} \sigma_{K}^{2}\right)}^{K}\right)_{0 \leq t \leq T}$, converges in probability, as $K \rightarrow \infty$, with respect to the Skorokhod topology on $\mathbb{D}([0, T], \mathrm{M}(\mathcal{X}))$ to the measure-valued process $\hat{\mathrm{n}}\left(x_{t}\right) \delta_{x_{t}}$, where $\left(x_{t}\right)_{0 \leq t \leq T}$ is given as a solution of the CEAD,

$$
\frac{d x_{t}}{d t}=\sum_{k=-A}^{A} k\left[k M\left(x_{t}\right) \hat{\mathrm{n}}\left(x_{t}\right) \partial_{1} f\left(x_{t}, x_{t}\right)\right]_{+} m\left(x_{t}, k\right), \quad \text { with initial condition } x_{0} .
$$

Remarks :

(i) The main result of the paper actually holds under weaker assumptions. More precisely, Assumption 3 can be replaced by the following :

Assumption $3^{\prime}$. The initial state $v_{0}^{K}$ has a.s. support $\left\{x_{0}\right\}$ with $x_{0} \in \mathcal{X}$ satisfying $\partial_{1} f\left(x_{0}, x_{0}\right) \neq 0$.

The reason is that, since $x \mapsto \partial_{1} f(x, x)$ is continuous, Assumption 3 is satisfied locally. Since moreover $x \mapsto \partial_{1} f(x, x)$ is Lipschitz-continuous, the CEAD never reaches in finite time an evolutionary singularity (i.e. a value $y \in \mathcal{X}$ such that $\left.\partial_{1} f(y, y)=0\right)$. In particular, for a fixed $T>0$, the CEAD only visits traits in some interval $I$ of $\mathcal{X}$ where $\partial_{1} f(x, x) \neq 0$. By modifying the parameters of the model out of $I$ in such a way that $\partial_{1} f(x, x) \neq 0$ everywhere in $\mathcal{X}$, we can apply Theorem 4.4 to this modified process $\tilde{\nu}$. Then, we deduce that $\tilde{\nu}_{t /\left(K u_{K} \sigma_{K}^{2}\right)}$ has support included in $I$ for $t \in[0, T]$ with high probability, and hence coincides with $\nu_{t /\left(K u_{K} \sigma_{K}^{2}\right)}$ on this time interval. 
(ii) The condition $q_{K} \ll \frac{\sigma_{K}^{1+\alpha}}{K \ln K}$ allows mutation events during an invasion phase of a mutant trait, but ensures that there is no "successful mutation" event during this phase.

(iii) The fluctuations of the resident population are of order $K^{-1 / 2}$, thus $K^{-1 / 2+\alpha}<<\sigma_{K}$ ensures that the sign of the initial growth rate is not influenced by the fluctuations of the population size. If a mutant trait $y$ appears in a monomorphic population with trait $x$, then its initial growth rate is $b-d(y)-\eta(y) C(y, x)\left\langle\nu_{t}^{K} \mid 1\right\rangle=$ $f(y, x)+o\left(\sigma_{K}\right)=(y-x) \partial_{1} f(x, x)+o\left(\sigma_{K}\right)$ since $y-x=O\left(\sigma_{K}\right)$.

(iv) $\exp \left(K^{\alpha}\right)$ is the time during which the resident population stays with high probability in an $O\left(\varepsilon \sigma_{K}\right)$ neighborhood of an attractive domain. This is a moderate deviation result. Thus, the condition $\exp \left(-K^{\alpha}\right) \ll$ $u_{K}$ ensures that the resident population is still in this neighborhood when a mutant occurs.

(vi) The time scale is $\left(K q_{K} \sigma_{K}^{2}\right)^{-1}$ since the expected time for a mutation event is $\left(K q_{K}\right)^{-1}$, the probability that a mutant invades is of order $\sigma_{K}$ and one needs $O\left(\sigma_{K}^{-1}\right)$ mutant invasions to see an $O(1)$ change of the resident trait value.

Still, such a strong filtering of mutations is probably the most questionable issue of realism concerning the modeling of evolution. The fluctuations around the deterministic system shall be extremely small and slightly deleterious mutations shall be well-separated for such conclusions to be satisfied. It seems unlikely that selective effects are so dominant even for rather large populations (a million of individuals or so). Especially since there is usually a structuring of the population in term of non-heritable or loosely heritable characteristics, that are not neutral for survival. Think for instance of the individual positions or their level of infection by parasites. As we have seen in Section 1, it may induce much more variability compared to the case where all the individuals are identical. In this time-scale of infinitesimal mutations, we may expect to see, in addition to these selective effect mainly driven by favorable mutations, also some noise due to the fixation of almost neutral mutations. The trait of the population is still quite likely to follow the direction given by invasion fitness, yet its displacement might be quite different from the one given by the CEAD and not as regular.

\section{Conclusion}

As we have seen, there is a large class of processes that can be rigorously obtained as limits of individual-based models appropriately rescaled. This is to be expected since this representation fits the closest to simulations of populations, with the minimal set of assumptions to include any interaction of interest. Nonetheless, the proof of convergence results are quite challenging and impose to be very specific on the way time-scales are well-separated. By the coupled observation both of the proofs and the simulations, the main weaknesses of the models usually appear much more salient.

Notably, we have evaluated the difficulty in estimating the birth rate in Section 1 from the sole knowledge of the trait at birth (because there is a lot of fluctuations until the birth event). In Sections 2 and 3 , the main issue appears to be that the predicted selection effects might be driven by too exceptional realizations of the stochastic process describing the dynamics of a typical individual. In simulations and actual life, such transitions from a very stable equilibrium to another one quite separated shall not happen exactly the way these models predict, notably for the time at which they occur. Although corrections can be made by some truncation, or by adding another term to the equation governing the density, the most realistic first step would possibly be to consider the Flemming-Viot representation introduced in Section 4. Yet, such measured-valued stochastic process is quite more challenging to describe. In Section 4, the assumption that mutations have only an infinitesimal effect appears difficult to combine with the fact that the population stays monomorphic and that the mutations are filtered depending on their effects.

This is the core of science to start with the most elementary models, like the ODE defining the growth rate of a population, and then to progressively incorporate more realistic features. With the current probabilistic tools at our disposal, it is clearly time to relate most of these models describing the dynamics of densities to the individual-based measure-valued processes. The main requirement is clearly that one averages over a large number of individuals, but this can be obtained in very various ways depending on the interactions of interest. 
In the case where a Central Limit Theorem holds, it can be exploited to confirm the stability of the less noisy dynamics. It may also provide another dynamics, a priori closer to IBM, with different qualitative properties as in Section 2. Moreover, the convergence results can be stated for very diverse time-scales, from the rapid adaptation of cells to the propagation of parasites and the evolution of species over millions of years. They provide an elementary way to unify the models of micro-biology, ecology and evolution. Thus, they enable to justify more rigorously the separation of the related time-scales or on the contrary motivate interesting couplings. Notably, in Section 2 the selective effects are closely linked to random demographic fluctuations; while in Section 4, the evolution of a marker as a measure-valued process is coupled in a very specific way to the punctual events of fixation for the traits under selection.

\section{Acknowledgment}

I wish to thank Etienne Pardoux, Viet Chi Tran and an anonymous reviewer for their contribution in improving the readability of this article. My thanks go also to Marc Hoffman and Benoit Henry for their answer.

\section{REFERENCES}

[1] Baar, M., Bovier, A.; The polymorphic evolution sequence for populations with phenotypic plasticity, Electron. J. Probab., Volume 23, no. 72, pp. 1-27 (2018)

[2] Baar, M., Bovier, A., Champagnat, N.; From stochastic, individual-based models to the canonical equation of adaptive dynamics in one step. Ann. Appl. Probab. 27, no. 2, 1093-1170. (2017)

[3] Bansaye, V., Delmas,J-F., Marsalle, L., Tran, V.; Limit theorems for Markov processes indexed by continuous time Galton-Watson trees Ann. Appl. Probab., V 21, Nbr 6, 2263-2314 (2011)

[4] Bansaye, V., Méléard, S.; Stochastic Models for Structured Populations, Scaling Limits and Long Time Behavior, Stochastics in Biological Systems, 1.4 (2015)

[5] Barton N.H., Etheridge A.M., Véber A.; The infinitesimal model: Definition, derivation, and implications. Theor Popul Biol.;V 118, pp. 50-73 (2017)

[6] Billiard, S., Collet,P., Ferrière, R., Méléard,S., Tran, V.; The effect of competition and horizontal trait inheritance on invasion, fixation and polymorphism. Journal of Theoretical Biology, Elsevier, 411, pp.48-58 (2016)

[7] Billiard, S., Ferrière, R., Méléard, S., Tran, V.; Stochastic dynamics of adaptive trait and neutral marker driven by ecoevolutionary feedbacks J. Math. Biol. 71: 1211-1242 (2015)

[8] Champagnat, N.; A microscopic interpretation for adaptative dynamics trait substitution sequence model. Stoch Process Appl 116:1127-1160 (2006)

[9] Champagnat, N., Ferrière, R., Méléard, S.; Unifying evolutionary dynamics: from individual stochastic processes to macroscopic models. Theoretical Population Biology, Elsevier, 69 (3), pp.297-321 (2006)

[10] Champagnat, N., Henry, B.; A probabilistic approach to Dirac concentration in non-local models of adaptation with several resources, Ann. Appl. Probab., V.29, N.4 (2019)

[11] Champagnat, N., Méléard, S.; Polymorphic evolution sequence and evolutionary branching. Probab Theory Relat Fields 151:45-94 (2011)

[12] Dawson, D.A.; Introductory Lectures on Stochastic Population Systems, Technical Report Series No. 451, Laboratory for Research in Stat. and Probab. (2010)

[13] Dawson, D. A.; Mesure-valued markov processes. v. 1541 of Lectures Notes in Math., Ecole d'Eté de probabilités de Saint-Flour XXI, Springer, pp 1-260, New York (1993)

[14] Diekmann, O., Jabin, P.E., Mischler, S., Perthame, B.; The dynamics of adaptation: An illuminating example and a HamiltonJacobi approach. Theor. Pop. Biol., 67:257-271 (2005)

[15] Durrett, R.; Probability Models for DNA Sequence Evolution, 2nd ed. Springer (2008)

[16] Etheridge, A.. An introduction to superprocesses, University Lecture Series, v. 20. American Mathematical Society, Providence (2000)

[17] Marc Hoffmann. Adaptive estimation in diffusion processes. Stochastic Process. Appl., 79(1):135-163 (1999)

[18] Hoffmann, M., Marguet, A.; Statistical estimation in a randomly structured branching population, Stochastic Process. Appl., to appear https://doi.org/10.1016/j.spa.2019.02.015 (2019)

[19] Kutoyants, Y. A.; Statistical inference for ergodic diffusion processes. Springer Science and Business Media (2013)

[20] Luo, S., Mattingly, J.; Scaling limits of a model for selection at two scales. Nonlinearity. 30. 10.1088/1361-6544/aa5499. (2015)

[21] Méléard, S., Tran, V.; Trait Substitution Sequence process and Canonical Equation for age-structured populations. J. Math. Biology, Springer (Germany), 58 (6), pp.881-921 (2009)

[22] J.A.J. Metz and al. Adaptative dynamics, a geometrical study of the consequences of nearly faithful reproduction. S.J. Van Strien and S.M. Verduyn Lunel (ed.), Stochastic and Spatial Structures of Dynamical Systems, 45:183-231 (1996) 
[23] Mirrahimi S., Barles G., Perthame B., Souganidis P. E.: Singular hamilton-jacobi equation for the tail problem. SIAM J. Math. Anal. 44(6), 4297-4319 (2012)

[24] Perthame, B., Gauduchon, M.; Survival thresholds and mortality rates in adaptive dynamics : conciliating deterministic and stochastic simulations. Math. Med. Biol. 27, 195-210 (2010)

[25] Sznitman, A.S.; Topics in propagation of chaos. In Ecole d'Eté de Probabilités de Saint-Flour XIX-1989, Lecture Notes in Math.1464, pp 165-251. Springer, Berlin (1991).

[26] Velleret, A.; Two level natural selection under the light of quasi-stationary distributions, on ArXiv : https://arxiv.org/abs/1903.10161 (2019).

[27] West, SA., Griffin, AS., Gardner A., Diggle SP. Social evolutiontheory for microorganisms. Nat. Rev. Microbiol. 4:597-607 (2006) 